NBER WORKING PAPER SERIES

\title{
USING ASSET PRICES TO MEASURE THE COST OF BUSINESS CYCLES
}

\author{
Fernando Alvarez \\ Urban J. Jermann \\ Working Paper 7978 \\ http://www.nber.org/papers/w7978 \\ NATIONAL BUREAU OF ECONOMIC RESEARCH \\ 1050 Massachusetts Avenue \\ Cambridge, MA 02138 \\ October 2000
}

We are grateful for the comments received from seminar participants at the Penn Macro Lunch at Wharton, the Federal Reserve of Philadelphia, Tom Sargent's reading group in Stanford, Carnegie-Mellon University, Duke University, Universidad Torcuato Di Tella, the 1999 LACEA meetings, UVA, UCLA, the NBER 2000 EFG meetings, Columbia University, University of Chicago, Princeton University, Yale University, the CEPR/ LIFE/ Weiss Center Conference in Vouliagmeni, the Banco de Portugal Conference in Oporto, and the SED meeting in Costa Rica. We thank especially Andy Abel, Bill Dupor, John Cachrane, Lars Hansen, Bob Lucas, and Chris Sims. Any views expressed here are ours and not necessarily those of the Federal Reserve Bank of Minneapolis, the Federal Reserve System, or the National Bureau of Economic Research.

(C) 2000 by Fernando Alvarez and Urban J. Jermann. All rights reserved. Short sections of text, not to exceed two paragraphs, may be quoted without explicit permission provided that full credit, including (C) notice, is given to the source. 
Using Asset Prices to Measure the Cost of Business Cycles

Fernando Alvarez and Urban J. Jermann

NBER Working Paper No. 7978

October 2000

JEL No. E32, G12

\section{$\underline{\text { ABSTRACT }}$}

We propose a method to measure the welfare cost of economic fluctuations that does not require full specification of consumer preferences and instead uses asset prices. The method is based on the marginal cost of consumption fluctuations, the per unit benefit of a marginal reduction in consumption fluctuations expressed as a percentage of consumption. We show that this measure is an upper bound for the benefit of reducing all consumption fluctuations. We also clarify the link between the cost of consumption uncertainty, the equity premium, and the slope of the real term structure. To measure the marginal cost of fluctuations, we fit a variety of pricing kernels that reproduce key asset pricing statistics. We find that consumers would be willing to pay a very high price for a reduction in overall consumption uncertainty. However, for consumption fluctuations corresponding to business cycle frequencies, we estimate the marginal cost to be about $0.55 \%$ of lifetime consumption based on the period 1889-1997 and about $0.30 \%$ based on 1954-97.

Fernando Alvarez

Department of Economics

University of Chicago

Social Science Building 422

1126 East $59^{\text {th }}$ Street

Chicago, IL 60637

and NBER

f-alvarez1@uchicago.edu
Urban J. Jermann

Research Department

Federal Reserve Bank of Minneapolis

90 Hennepin Avenue

Minneapolis, MN 55480-0291

and Wharton School, Univ. of Pennsylvania

and NBER

jermann@wharton.upenn.edu 


\section{Introduction}

In a seminal contribution, Lucas (1987) proposes a measure of the welfare cost of economic fluctuations. His measure is defined as the compensation required to make the representative agent indifferent between consumption plans with and without business cycle fluctuations. With this measure, Lucas finds a small cost of business cycles. Subsequently, several studies have proposed estimates of this cost of business cycles under alternative assumptions on preferences and the consumption processes. Primarily as a function of the specification and parameterization of preferences, these estimates vary widely across studies. ${ }^{1}$ In this paper, we measure the welfare cost of business cycles without fully specifying consumer preferences; instead, we directly use financial market data. We find that by directly measuring the premia for aggregate risk, we circumvent some of the difficulties related to specifying a utility function.

We start by generalizing Lucas' cost of business cycles along two dimensions. First, we derive the marginal cost of consumption fluctuations. Lucas' cost of business cycles measures the welfare gain from removing all the business cycle risk; it can be thought of as a total cost. We define a cost that measures the welfare benefits from reduced fluctuations at the margin. This definition has two useful features: (1) Because it is a marginal cost, we can use asset prices to estimate the cost of business cycles for a representative agent. (2) Given that most economic policies would not be intended to eliminate business cycle fluctuations entirely, knowing the potential benefits at the margin may be useful in itself. Our second dimension of generalization concerns the type of consumption fluctuations that one associates with business cycles. Lucas' consumption is subject to only temporary deviations from a deterministic trend. Several later studies specify consumption as a nonstationary process (for instance, Obstfeld (1994) and Campbell and Cochrane (1995)), while the cost of business cycles continues to be measured as the gain from eliminating consumption uncertainty entirely, including the risks

\footnotetext{
${ }^{1}$ Lucas' estimates are well below $1 \%$ of consumption. Obstfeld (1994) finds slightly higher costs than Lucas when allowing for stochastic growth trends and preferences that distinguish between risk aversion and intertemporal elasticity of substitution. Campbell and Cochrane (1995) find larger costs of business cycles by using habit-formation preferences that are also able to explain the equity premium. Atkeson and Phelan (1994) present an example of an incomplete markets economy that can generate the equity premium, but nevertheless has only a small cost of business cycles. Other examples include: Dolmas (1998); Hansen, Sargent, and Tallarini (1999); Krusell and Smith (1999); Otrok (1998); Tallarini (2000); for related literature about the welfare gains from international integration see Lewis (1996) and Van Wincoop (1999).
} 
associated with the permanent stochastic components. Our definition is general enough to encompass this case, but also allows for business cycle fluctuations to be defined differently, for instance, as the cyclical consumption volatility excluding a possibly stochastic trend. We find this distinction useful because a priori one may think that some policies affect primarily economic behavior at business cycle frequencies without affecting the trend behavior of the economy. Even without this distinction about the source of economic fluctuations, it may be interesting to know the costs of the more short-lived cyclical fluctuations relative to the costs of overall consumption uncertainty.

Before presenting estimates of our measure of the welfare cost of business cycles, we derive some analytical results. Under general specification, we show that the marginal cost of reducing fluctuations is higher than the total cost of these fluctuations; hence, our estimates are upper bounds. For more specific cases, such as expected discounted utility, we obtain a sharper bound where the marginal cost of consumption uncertainty is twice the total cost. The marginal cost is also an upper bound with consumption externalities of a general class that we label envy.

Several of the above-cited studies highlight the relationship between Lucas' low cost of business cycles and the equity premium puzzle. In particular, studies with preference specifications that can solve the equity premium puzzle usually report a cost of business cycles larger than Lucas' original estimate. We show that the marginal cost of consumption uncertainty is not equal to the (consumption) equity premium in general. The equity premium is the excess return of equity over a riskfree rate of return for a given holding period. In contrast, the marginal cost of consumption uncertainty is equal to the excess in the price of a perpetual bond with growing coupons over the price of a consumption equity claim. Two of the factors that make the marginal cost of consumption uncertainty different from the equity premium are the steepness of the term structure and the persistence of the shocks. We clarify the link between the two and provide examples where the quantitative differences are of first-order importance.

Motivated by our finding that the cost of consumption uncertainty can be expressed as the ratio of the prices of two assets, we derive a simple formula for the marginal cost. The marginal cost can be written as a function of long-term real yields, aggregate dividend/price ratios, and an estimate of the economy's trend growth rate. If consumption were equal to dividends, this formula could be used to measure the cost of business cycles. Strictly speaking, however, it is a measure of the cost associated with all the uncertainty about aggregate dividends. Measuring the cost of dividend uncertainty in this way, we find that the cost amounts to $619 \%$ 
of the level of consumption based on a sample period of $1889-1997$ and $173 \%$ for the postwar period of 1954-97.

To relax the assumption of our simplified expression for the marginal cost and to better measure the relevant prices, we estimate an asset pricing kernel. We estimate a pricing kernel as a way to interpolate from the available asset prices the prices for the assets that measure the cost of consumption fluctuations. Our asset pricing kernel is consistent with U.S. historical returns on equity, the term structure, and the price/dividend ratios, all features that we show, on theoretical grounds, are important for measuring the cost of business cycles. In the state space for the pricing kernel, we include variables that determine the market price of risk in different popular models. Since consumption is an element of the state space, our pricing kernel allows us to differentiate consumption from dividends. We model consumption and the pricing kernel as non-stationary. This choice has quantitative implications for some of our measures. Our choice is motivated by a bound, in the spirit of the Hansen-Jagannathan bound, developed in Alvarez and Jermann (2000). It implies that the pricing kernel cannot be stationary and explain simultaneously the historical real term structure and the equity premium.

Our definition of business cycles is based on a frequency domain approach, with business cycles corresponding to cycles of at most 8 . Our preferred filter is derived through an ad hoc adjustment to a one-sided band-pass filter so as to match the volatility of the deviations from trend obtained with a more accurate two-sided filter. ${ }^{2}$ Based on this, we estimate the marginal cost to be about $0.55 \%$ of lifetime consumption for the period $1889-1997$ and about $0.30 \%$ for the period 1954-97. We also estimate the cost of all consumption uncertainty, that is, including cycles of any length. We find very large costs. With cointegrated consumption and dividends, in many cases, costs are around $30 \%$ of lifetime consumption; without cointegration, these costs amount in general to several hundred percent. Finally, we link these estimates to the benefit of increasing growth-a small modification of our framework allows us to do this in a consistent way. The benefit from stabilization in terms of additional long-term growth is shown to be equal to the marginal cost expressed as a percentage of consumption divided by the duration of an equity claim with consumption as payouts. We find that eliminating business cycle fluctuations is worth less than 1 basis point of additional growth. Eliminating all consumption uncertainty is valued at roughly $1 \%$ of additional growth.

As a way to check our quantitative results, and to provide intuition, we also

\footnotetext{
${ }^{2}$ As becomes clear below, our approach requires the use of a one-sided filter.
} 
examine the special case where the logarithms of consumption and the pricing kernel follow random walks. We derive a simple expression for the marginal cost of consumption fluctuations. The marginal cost is approximately equal to the excess return of an equity claim to consumption multiplied by a factor that is a simple function of the moving-average coefficients used to define the business cycle components. Estimates of this simplified expression, where the pricing kernel is only required to fit the excess returns of different CRSP portfolios, yield findings that are similar to the ones obtained with the more general specification.

The paper is organized as follows. Section 2 defines the marginal cost of consumption fluctuations. Section 3 compares marginal and total costs. Section 4 studies the relationship between the marginal cost of consumption uncertainty and the equity premium. Section 5 presents our empirical estimates of the marginal cost of business cycles. Section 6 compares the cost of fluctuations with the effects of growth. Section 7 concludes. Appendix A describes the data used, and Appendix B contains the proofs of propositions.

\section{Defining the Marginal Cost of Consumption Fluctuations}

We start by defining our measures of the cost of business cycles. We generalize Lucas' definition along two dimensions. First, allowing for partial reduction in consumption fluctuations, we define a total cost function, and as its derivative evaluated at the point where the consumers bear all the consumption fluctuations, we define the marginal cost of consumption fluctuations. As a second dimension of generalization, we leave open for the moment which type of consumption fluctuations are eliminated. As we discuss more in detail in the following sections, we eliminate, for instance, all consumption uncertainty or only cyclical consumption fluctuations for some frequencies.

The analysis is carried out under the assumptions of a representative agent economy. In each period $t$, the economy experiences one of finitely many events $z_{t} \in Z$. We denote by $z^{t}=\left(z_{0}, z_{1}, \ldots, z_{t}\right)$ the history of events up through and including period $t$. We index consumption by histories, so we write $C: \mathbf{Z} \rightarrow R_{+}$, where $\mathbf{Z} \equiv \prod_{t \geq 1} Z^{t}$, or simply $\{C\}=\left\{C_{t}\left(z^{t}\right): \forall t \geq 1, z^{t} \in Z^{t}\right\}$.

Definition 2.1. We define the total cost of consumption fluctuations function $\Omega(\alpha)$ as the solution of

$$
U((1+\Omega(\alpha))\{C\})=U((1-\alpha)\{C\}+\alpha\{\mathfrak{C}\}),
$$


where $\alpha \in[0,1], C: \mathbf{Z} \rightarrow R_{+}$denotes the process of consumption, and $\mathfrak{C}: \mathbf{Z} \rightarrow$ $R_{+}$denotes the process for the trend starting in period 1 . Here $U(\cdot)$ is a utility function, mapping consumption processes into $R$.

The scalar $\alpha$ measures the fraction of risky consumption $C$ that has been replaced by the less risky trend consumption $\mathfrak{C}^{3}$ The total cost function gives the total benefit from reducing consumption fluctuations as a function of the fraction of the reduction in fluctuations. It is straightforward to see that $\Omega(0)=0$, so that no reduction in fluctuations generates no benefit.

The next definition serves mainly notational convenience. We define the total cost of consumption fluctuations $\omega$, as $\omega \equiv \Omega(1)$, or equivalently $U((1+\omega)\{C\})=$ $U(\{\mathfrak{C}\})$. As a particular case, we define the trend consumption to be $\{\mathfrak{C}\}=$ $\left\{E_{0}(C)\right\}$, that is where $\mathfrak{C}\left(z^{t}\right)=E_{0}\left(C_{t}\right)$ for all $t$ and $z^{t}$. In this case, we have that

$$
U((1+\omega)\{C\})=U\left(\left\{E_{0}(C)\right\}\right)
$$

which is Lucas' definition of the cost of business cycles. Thus, Lucas' definition can be seen as the total benefit associated with eliminating all the consumption fluctuations, that is, $\alpha=1$, and where consumption fluctuations are defined as consumption uncertainty, that is, resulting in the exchange of consumption for its expected path. When we use this specification for $\mathfrak{C}$ we refer to $\Omega$ as the cost of consumption uncertainty, since $\mathfrak{C}$ is deterministic, to distinguish the specification from the more general case, where we refer to $\Omega$ as the cost of consumption fluctuations.

Note that the specification in equation (2.2) differs slightly from Lucas' and the literature's standard specification because we choose to begin compensation as of $t=1$; the standard has been to start compensation at $t=0$. We choose this departure because our definition is more consistent with the idea of ex-dividend security prices, some of our qualitative results present themselves more tractably with our definition, and the quantitative difference between the standard definition and ours will be insignificant.

For the next definition we assume that $U$ is differentiable with respect to each $C_{t}\left(z^{t}\right)$ for all $t$ and $z^{t}$. We denote the partial derivatives by $U_{z^{t}}(\{C\}) \equiv$ $\partial U(\{C\}) / \partial C_{t}\left(z^{t}\right)$.

\footnotetext{
${ }^{3}$ Our use of the word trend does not imply any statistical statement. It is simply a label for a consumption process that is different from actual consumption and presumably less risky.
} 
Definition 2.2. We define the marginal cost of consumption fluctuations $\omega^{m}$ as the derivative of the total cost function $\Omega$ evaluated at $\alpha=0$, that is,

$$
\omega^{m} \equiv \Omega^{\prime}(0)=\frac{\sum_{t=1}^{\infty} \sum_{z^{t}} U_{z^{t}}(\{C\}) \cdot\left[\mathfrak{C}\left(z^{t}\right)-C_{t}\left(z^{t}\right)\right]}{\sum_{t=1}^{\infty} \sum_{z^{t}} U_{z^{t}}(\{C\}) \cdot C_{t}\left(z^{t}\right)} .
$$

Thus, $\omega^{m}$ measures the per unit benefit of a marginal reduction in consumption fluctuations expressed as a percentage of consumption. This measure can be considered as the market price of consumption fluctuations. ${ }^{4}$

For any process $X: \mathbf{Z} \rightarrow R$, define $V_{0}[\{X\}]$ as follows:

$$
V_{0}[\{X\}] \equiv \sum_{t=1}^{\infty} \sum_{z^{t}} U_{z^{t}}(\{C\}) \cdot X_{t}\left(z^{t}\right) .
$$

This is the shadow price, for the representative agent, of an asset with payouts given by $\{X\}$. Under this convention, it is immediately seen that

$$
\omega^{m}=\frac{V_{0}[\{\mathfrak{C}\}]}{V_{0}[\{C\}]}-1 .
$$

Thus, one can interpret the marginal cost of consumption fluctuations as a ratio of the values of two securities: a claim to the consumption trend, $V_{0}[\{\mathfrak{C}\}]$, and a claim to consumption, $V_{0}[\{C\}]$, or equivalently, a consumption-equity claim.

These generalizations of Lucas' cost of business cycles have some attractive features. First, focusing on a marginal cost, we can hope to measure this cost by using information on the representative agent's marginal evaluation contained in security prices. Second, we can now think of the benefits of partial reduction in consumption fluctuations. Moreover, as our analysis in the next section shows, we can use our measure of the marginal cost to bound the standard total cost of business cycles for a large class of preference specifications, without the need to fully parameterize these preferences.

\section{Comparing Marginal Cost and Total Cost of Consump- tion Fluctuations}

The marginal cost defined in Section 2 can be measured using asset prices, while the total cost requires a fully specified utility function. In this section, we show

\footnotetext{
${ }^{4}$ For a discussion about the thought experiment underlying this definition, see Section 3.3., where we discuss the cost of consumption fluctuations with consumption externalities.
} 
that under some assumptions, it is possible to gain information about the total cost once we know the marginal cost. Alternatively, the marginal cost may be interesting in itself, since some policies may only move partially in the direction of eliminating fluctuations. For instance, if most of the benefits from stabilization occur already for low values of $\alpha$, then implementing a policy that moves in this direction - even if it does not make consumption equal to the trend value - will be worthwhile.

The main results of this section are the following. First, the concavity of $U$ implies that $\Omega$ is concave for small $\alpha$, hence, the marginal cost $\omega^{m}$ is an upper bound for $\Omega(\alpha) / \alpha$ for small $\alpha$. Second, if $U$ is concave and homothetic, so that the total cost does not depend on the scale of the economy, then the total cost $\Omega(\alpha)$ is concave, so that the marginal cost is an upper bound for the total cost, that is, $\omega^{m}=\Omega^{\prime}(0) \geq \omega=\Omega(1)$. Many preference specifications used in quantitative asset pricing studies satisfy this assumption, since many researchers impose the same form of scale invariance. Third, we consider the case when $U$ is given by the expected value of a concave utility and the trend is given by the expected value of consumption. In this case, $\Omega$ is concave not only for $\alpha$ close to zero, but also for $\alpha$ close to one. If in addition we have time-separable utility, we show that for small variance, $\omega=\frac{1}{2} \omega^{m}$, and the total cost is given by the insurance risk premium.

We make the following initial assumptions: $U(\{C\})$ is increasing and concave in $\{C\}$. We also assume that the process $\{\mathfrak{C}\}$ is preferred to $\{C\}$, that is, $U(\{\mathfrak{C}\})>U(\{C\})$. Under these conditions, it is straightforward to see that $\Omega(\alpha) \geq 0$. Our first result is that $\Omega$ is concave for small $\alpha$.

Proposition 3.1. Let $U$ be increasing, concave, and twice differentiable. Then $\Omega^{\prime \prime}(0) \leq 0$.

This proposition implies for small $\alpha$ that $\omega^{m} \equiv \Omega^{\prime}(0) \geq \frac{\Omega(\alpha)}{a}$; that is, the marginal cost, $\omega^{m} \equiv \Omega^{\prime}(0)$, is larger than the average cost, $\frac{\Omega(\alpha)}{a}$.

\subsection{Homothetic preferences and scale-free cost functions}

If we require that the cost of fluctuations $\Omega(\alpha)$ be the same for the processes $\{C\}$ and $\{\mathfrak{C}\}$ as for the processes $\{\lambda C\}$ and $\{\lambda \mathfrak{C}\}$, where $\lambda$ is any positive scalar, then we must impose some additional restrictions on the utility function $U$. This requirement implies that the cost of consumption fluctuations will not differ merely because economies are rich and poor. Specifically, we require $U$ to be homothetic; 
that is, $U$ is homogeneous of degree $1-\gamma$, i.e., for any positive scalar $\lambda>0$, and for $\lambda\{C\}$ defined as $\lambda\{C\}_{t}\left(z^{t}\right)=\lambda C_{t}\left(z^{t}\right)$ for each $z^{t}$, we have

$$
U(\lambda\{C\})=\lambda^{1-\gamma} U(\{C\})
$$

Under this assumption, we obtain that the marginal cost is higher than the total cost.

Proposition 3.2. Assume that $U$ is increasing, concave, and homothetic. Also assume that $\{\mathfrak{C}\}$ is preferred to $\{C\}$, that is, $U(\{\mathfrak{C}\})>U(\{C\})$. Then $\Omega(\alpha)$ is concave, and thus,

$$
\omega^{m} \equiv \Omega^{\prime}(0) \geq \Omega(1) \equiv \omega
$$

Examples from the literature that satisfy this homogeneity property are the preferences used in Abel (1999), Epstein and Zin (1991), Mehra and Prescott (1985), and Tallarini (2000). Some utility functions with additive habit $\longrightarrow$ such as those in Campbell and Cochrane (1995), Constantinides (1990) and Jermann (1998) - do not satisfy this property for a predetermined habit level. ${ }^{5}$

\subsection{The cost of all uncertainty with expected utility}

Now we present some implications for the total and marginal cost $\Omega$ and $\Omega^{\prime}$ when the utility $U$ is given by expected utility. We also assume that the trend $\{\mathfrak{C}\}$ is given by the expected value of consumption; that is, we evaluate the elimination of all uncertainty.

Let $U$ be given by the expected value of a function $u: R_{+}^{\infty} \rightarrow R$. This specification allows time nonseparabilities, including habit formation or durability, for instance. Notice that if $u$ is concave, then $U$ is concave; and hence, from our previous proposition, $\Omega$ is concave for $\alpha$ close to zero. Now we obtain a complementary result, by showing that $\Omega$ is concave for values of $\alpha$ close to 1 .

Proposition 3.3. Let $U$ be given by the expected value of a concave and differentiable function $u$, and let $\mathfrak{C}_{t}\left(z^{t}\right)=E_{0}\left(C_{t}\right)$ for all $z$ and $t$. Then $\Omega^{\prime}(1)=0$ and $\Omega(\alpha)$ is concave for $\alpha$ close to one.

\footnotetext{
${ }^{5}$ If the habit level is a function of only a few lags and when there is some separability across time, such as in Jermann (1998), it can be shown that a result similar to Proposition 3.2 holds after a suitable restatement of the proposition.
} 
Now we further specialize $u$ by eliminating time non-separabilities and by considering the case where consumption fluctuations are small. We show that for an approximation up to the order of the variance of consumption, the total cost of uncertainty equals half of the marginal cost; that is, $\Omega(1)=1 / 2 \Omega^{\prime}(0)$. In this case, the marginal cost is given by a weighted average of the product of risk aversion and the variance of consumption for different periods. We also consider a higher order approximation to examine the role of skewness in consumption fluctuations. We show that if the period utility function $u$ displays prudence, that is $u^{\prime \prime \prime}>0$, and if consumption fluctuations have negative skewness, then we obtain a stronger inequality, that is $\Omega(1)<\frac{1}{2} \Omega^{\prime}(0){ }^{6}$

Consider first the one-period case, where consumption is given by

$$
C=\bar{C}(1+\sigma \varepsilon)
$$

for a zero-mean random variable $\varepsilon$. The parameter $\sigma$ indexes the amount of risk. The trend is given by the expected value, that is, $\mathfrak{C}=\bar{C} \equiv E[C]$. Notice that the variance of $C$ is proportional to $\sigma^{2}$-that is, $\operatorname{var}(C / \bar{C})=\sigma^{2} E \varepsilon^{2}$-and that its third moment is proportional to $\sigma^{3}$. We include $\sigma$ as an argument of the total and the marginal costs, which are given by

$$
\begin{gathered}
E[u(C(1+\Omega(1, \sigma)))] \equiv E[u(\bar{C}(1+\sigma \varepsilon)(1+\Omega(1, \sigma)))]=u(\bar{C}) \\
\Omega^{\prime}\left(0, \sigma^{2}\right)=\frac{E\left[u^{\prime}(C)(\bar{C}-C)\right]}{E\left[u^{\prime}(C) C\right]} \equiv \frac{-E\left[u^{\prime}(\bar{C}+\bar{C} \sigma \varepsilon)(\bar{C} \sigma \varepsilon)\right]}{E\left[u^{\prime}(\bar{C}+\bar{C} \sigma \varepsilon)(\bar{C}+\bar{C} \sigma \varepsilon)\right]} .
\end{gathered}
$$

Proposition 3.4. If $E\left[u^{\prime \prime \prime \prime}(\bar{C}(1+\varepsilon)) \varepsilon^{4}\right]$ is finite, then

$$
\begin{aligned}
& \Omega(1, \sigma)=\frac{1}{2}\left[-\frac{\bar{C} u^{\prime \prime}(\bar{C})}{u^{\prime}(\bar{C})} \sigma^{2} E \varepsilon^{2}-\frac{1}{3} \frac{\bar{C}^{2} u^{\prime \prime \prime}(\bar{C})}{u^{\prime}(\bar{C})} \sigma^{3} E \varepsilon^{3}\right]+o\left(\sigma^{3}\right), \\
& \Omega^{\prime}(0, \sigma)=\left[-\frac{\bar{C} u^{\prime \prime}(\bar{C})}{u^{\prime}(\bar{C})} \sigma^{2} E \varepsilon^{2}-\frac{1}{2} \frac{\bar{C}^{2} u^{\prime \prime \prime}(\bar{C})}{u^{\prime}(\bar{C})} \sigma^{3} E \varepsilon^{3}\right]+o\left(\sigma^{3}\right),
\end{aligned}
$$

where $h(\sigma)=f(\sigma)+o\left(\sigma^{p}\right)$ means that $\lim _{\sigma \rightarrow 0}[h(\sigma)-f(\sigma)] / \sigma^{p}=0$.

\footnotetext{
${ }^{6}$ Rietz (1988) assumes that there is a small probability of a large drop in consumption, motivated by the Great Depression, and he shows that this leads to a substantial increase in the equity premium.
} 
Notice that these expressions imply that

$$
\Omega^{\prime}(0, \sigma)=2 \Omega(1, \sigma)-\frac{\sigma^{3}}{6} \frac{\bar{C}^{2} u^{\prime \prime \prime}(\bar{C})}{u^{\prime}(\bar{C})} E \varepsilon^{3}+o\left(\sigma^{3}\right) .
$$

Thus, ignoring all the terms smaller than $\sigma^{2}$, the marginal cost is twice the total cost, $\Omega^{\prime}(0, \sigma)=2 \Omega(1, \sigma)$. Furthermore, considering higher moments, and under the assumptions that $u^{\prime \prime \prime}(\bar{C})>0$ and $E \varepsilon^{3}<0$, then we obtain that $\Omega^{\prime}(0, \sigma)>$ $2 \Omega(1, \sigma)$.

The next example illustrates these approximations for a distribution with an approximately zero third moment.

Example 3.5. Let $u$ be given by a utility with constant relative risk aversion $\gamma$, that is $u(C)=\frac{C^{1-\gamma}}{1-\gamma}$, and let $\log C$ be distributed as $N\left(\mu, \hat{\sigma}^{2}\right)$. Recall that if $C$ is log-normal, then

$$
\operatorname{var}(C / \bar{C}) \equiv \frac{\operatorname{var}(C)}{[E(C)]^{2}}=\left(e^{\hat{\sigma}^{2}}-1\right)=\hat{\sigma}^{2}+o\left(\hat{\sigma}^{3}\right) .
$$

Simple computations show that,

$$
\begin{aligned}
& \Omega(1, \sigma)=e^{\frac{1}{2} \gamma \hat{\sigma}^{2}}-1=\frac{1}{2} \gamma \hat{\sigma}^{2}+o\left(\hat{\sigma}^{3}\right), \\
& \Omega^{\prime}(0, \sigma)=e^{\gamma \hat{\sigma}^{2}}-1=\gamma \hat{\sigma}^{2}+o\left(\hat{\sigma}^{3}\right) .
\end{aligned}
$$

Note that, in this example, for small $\sigma, \sigma \varepsilon \cong \log C$, which is normally distributed, and hence, it has a zero third moment.

Now we consider the multiperiod case, where

$$
U(\{C\})=\sum_{t=1}^{T} \beta^{t} E_{0}\left[u\left(C_{t}\right)\right]
$$

with $C_{t}=\bar{C}_{t}\left(1+\sigma \varepsilon_{t}\right)$, with $\varepsilon_{t}$ a random variable satisfying $E_{0}\left[\varepsilon_{t}\right]=0$, and with time-discount factor $\beta$. We keep the assumption that $\mathfrak{C}_{t}=\bar{C}_{t} \equiv E_{0}\left[C_{t}\right]$. The next proposition says that expressions for the cost in the multiperiod case are equal to a weighted average of those for the one-period case. 
Proposition 3.6. If

$$
\beta^{t} E_{0}\left[\frac{\bar{C}_{t}^{2} u^{\prime \prime \prime \prime}\left(\bar{C}_{t}\left(1+\varepsilon_{t}\right)\right)}{u^{\prime}\left(\bar{C}_{t}\right)} \varepsilon_{t}^{4}\right]
$$

is finite, then

$$
\begin{aligned}
& \Omega(1, \sigma)=\sum_{t=1}^{T} \bar{w}_{t}\left\{-\frac{1}{2} \frac{\bar{C}_{t} u^{\prime \prime}\left(\bar{C}_{t}\right)}{u^{\prime}\left(\bar{C}_{t}\right)} \sigma^{2} E_{0} \varepsilon_{t}^{2}-\frac{1}{6} \frac{\bar{C}_{t}^{2} u^{\prime \prime \prime}\left(\bar{C}_{t}\right)}{u^{\prime}\left(\bar{C}_{t}\right)} \sigma^{3} E_{0} \varepsilon_{t}^{3}\right\}+o\left(\sigma^{3}\right), \\
& \Omega^{\prime}(0, \sigma)=\sum_{t=1}^{T} \bar{w}_{t}\left\{-\frac{\bar{C}_{t} u^{\prime \prime}\left(\bar{C}_{t}\right)}{u^{\prime}\left(\bar{C}_{t}\right)} \sigma^{2} E_{0} \varepsilon_{t}^{2}-\frac{1}{2} \frac{\bar{C}_{t}^{2} u^{\prime \prime \prime}\left(\bar{C}_{t}\right)}{u^{\prime}\left(\bar{C}_{t}\right)} \sigma^{3} E_{0} \varepsilon_{t}^{3}\right\}+o\left(\sigma^{3}\right),
\end{aligned}
$$

where the weights $\bar{w}_{t}$ are defined as

$$
\bar{w}_{t}=\frac{\beta^{t} u^{\prime}\left(\bar{C}_{t}\right) \bar{C}_{t}}{\sum_{j=1}^{\infty} \beta^{j} u^{\prime}\left(\bar{C}_{j}\right) \bar{C}_{j}} .
$$

Notice that this proposition implies that

$$
\Omega^{\prime}(0, \sigma)=2 \Omega(1, \sigma)-\sum_{t=1}^{T} \bar{w}_{t}\left\{\frac{\sigma^{3}}{6} \frac{\bar{C}_{t}^{2} u^{\prime \prime \prime}\left(\bar{C}_{t}\right)}{u^{\prime}\left(\bar{C}_{t}\right)} E_{0} \varepsilon_{t}^{3}\right\}+o\left(\sigma^{3}\right) .
$$

Thus if we neglect the terms of order higher than $\sigma^{2}, \Omega^{\prime}(0, \sigma)=2 \Omega(1, \sigma)$. The following example uses this approximation for a CRRA utility function.

Example 3.7. Let $u$ be a utility function with constant relative risk aversion $\gamma$, so that $u(C)=C^{1-\gamma} /(1-\gamma)$. In this case, neglecting the terms of order higher than $\sigma^{3}$, we see that

$$
\begin{aligned}
\Omega^{\prime}(0, \sigma) & =\sum_{t} \bar{w}_{t}\left\{\gamma \sigma^{2} E_{0} \varepsilon_{t}^{2}-\frac{1}{2} \gamma(1+\gamma) \sigma^{3} E_{0} \varepsilon_{t}^{3}\right\}, \\
\Omega(1, \sigma) & =\sum_{t} \bar{w}_{t}\left\{\frac{1}{2} \gamma \sigma^{2} E_{0} \varepsilon_{t}^{2}-\frac{1}{6} \gamma(1+\gamma) \sigma^{3} E_{0} \varepsilon_{t}^{3}\right\} .
\end{aligned}
$$

We end this section with a numerical example that illustrates the accuracy of the approximation for small $\sigma^{2}$. In each panel of Figure 1, we plot the total cost, $\Omega(\alpha)$, and the marginal cost, $\Omega^{\prime}(\alpha)$, as a function of the fraction of risk removed, $\alpha$. In the three panels, the distribution of consumption has the same mean and 
variance, but the skewness is different. In particular, the standard deviation is $3.6 \%$, the mean 1 , and $u$ is given by a CRRA function with risk aversion $\gamma=10$. We introduce skewness by making downward moves four times as large a upward moves for negative skewness and vice versa for positive skewness. Notice that, as Proposition 3.4 says, the total costs are very close to half of the value of the marginal costs - indicated by the dotted lines - that is, $\frac{1}{2} \omega^{m}=\frac{1}{2} \Omega^{\prime}(0) \approx \Omega(1)$ $=\omega$. Our general results about the impact of third moments are also clearly illustrated. In particular, in the second panel, where skewness is negative, the total cost is smaller than half of the marginal cost, which is what Proposition 3.4 says for utility functions, such as CRRA, for which $u^{\prime \prime \prime}>0$. Thus, negative skewness of consumption makes the marginal cost larger relative to the total cost The third panel shows that for positive skewness, the opposite holds. In a related comparison, Hansen, Sargent, and Tallarini (1999) compute local and global mean-risk trade-offs for consumers with risk-sensitive preferences; they find their local measure to be between two and four times higher than the global one.

\subsection{The cost of fluctuations with consumption externalities}

Up to now, we have considered the case of a representative agent economy without consumption externalities. Now we define aggregate consumption externalities by including aggregate consumption $\{\bar{C}\}$ in the utility function as a separate argument, together with the agent's consumption $\{C\}$, so that utility is given by $U(\{C\},\{\bar{C}\})$.

With externalities, the interpretation of the compensation $\Omega$ depends on the exact nature of the experiment. In particular, there are two ways of thinking about reducing fluctuations in consumption. One experiment is to stabilize consumption of only one agent, keeping aggregate consumption unchanged. Another experiment is to stabilize consumption of all the agents. In the first experiment, the total cost function is given by

$$
U((1+\Omega(\alpha))\{C\},\{C\})=U(\alpha\{C\}+(1-\alpha)\{\mathfrak{C}\},\{C\}) .
$$

Then the benefit of a small reduction in fluctuations can be measured by the corresponding marginal cost. As explained above, the marginal cost $\Omega^{\prime}(0)$ equals the ratio of the prices of two securities, as in (2.4). Here $U_{z^{t}}(\{C\})$ is replaced by $U_{z^{t}}(\{C\},\{\bar{C}\})$ defined as

$$
U_{z^{t}}(\{C\},\{\bar{C}\}) \equiv \frac{\partial U(\{C\},\{\bar{C}\})}{\partial C_{t}\left(z^{t}\right)}
$$


In the second experiment, the total cost function $\tilde{\Omega}$ is defined as

$$
U((1+\tilde{\Omega}(\alpha))\{C\},\{C\})=U(\alpha\{C\}+(1-\alpha)\{\mathfrak{C}\}, \alpha\{C\}+(1-\alpha)\{\mathfrak{C}\})
$$

The function $\tilde{\Omega}$ differs from $\Omega$, since it includes the effect of the externality. However, because market prices do not internalize the effect of aggregate consumption, they do not equal $\tilde{\Omega}^{\prime}(0)$.

While consumption externalities can have various forms, the consumption externalities used to account for the determinants of aggregate risk are of a particular type. We call envy a general type of externality that is related to the ones used in the literature, such as the "catching up with the Joneses" in Abel (1999), and the "external habit" in Campbell and Cochrane (1995).

Definition 3.8. We say that the representative agent exhibits envy if, for any $C$ and $\hat{C}$ such that

$$
U(\{\hat{C}\},\{C\}) \geq U(\{C\},\{C\})
$$

then

$$
U(\{\hat{C}\},\{C\}) \geq U(\{\hat{C}\},\{\hat{C}\}) \text {. }
$$

The interpretation of this definition is that agents individually use the consumption of the representative agent as a benchmark for their own consumption. The next proposition shows that if the representative agent exhibits envy, then the total benefits from stabilizing everybody's consumption are even smaller than the benefits from stabilizing one person's consumption.

Proposition 3.9. Assume that the trend consumption $\{\mathfrak{C}\}$ is such that

$$
U(\{\mathfrak{C}\},\{C\})>U(\{C\},\{C\}) .
$$

If $U$ is increasing in its first argument and concave, and if Definition 3.8 holds, then

for $\alpha \in[0,1]$.

$$
\Omega(\alpha) \geq \tilde{\Omega}(\alpha)
$$

As a corollary of Proposition 3.9, if $U\{\cdot, C\}$ has the properties of $U(\cdot)$ in Proposition 3.2, then

$$
\omega^{m} \equiv \Omega^{\prime}(0) \geq \Omega(1) \geq \tilde{\Omega}(1) .
$$

Thus, the ratio of the market prices of the securities in equation (2.4) is an upper bound for the total cost of fluctuations in both experiments, that is, whether consumption is stabilized for one agent or for all agents. 


\section{Comparing the Marginal Cost of Consumption Uncer- tainty with the Equity Premium}

It seems widely recognized that there is a relationship between the equity premium and the cost of consumption uncertainty. Indeed, both are measures of the compensation required to bear aggregate risk. To our knowledge, however, no detailed comparison of these measures has been made in the literature. In this section, we make that comparison. To facilitate it, we select an equity which has dividends equal to consumption; that is, we focus on consumption-equity. Our analysis clearly exposes the differences, conceptually and quantitatively, between the marginal cost of consumption uncertainty and the equity premium. Our analysis also provides insights into how to extract information contained in the equity premium to learn about the cost of business cycles.

We approach the comparison from two complementary angles. First, in Section 4.1, we use a decomposition into the fundamental risk components inspired by Campbell (1986) and Jermann (1998). This characterization has two important implications for estimating the marginal cost using asset price data. One implication is that the slope of the term structure is an important determinant for the equity premium, but it is not a determinant for the marginal cost. The steeper the term structure is, the larger the equity premium can be, relative to the marginal cost of uncertainty. The other implication is that the degrees of persistence of the pricing kernel and of the consumption processes have different impacts on the marginal cost and on the equity premium. The more persistent these processes are, the larger the marginal cost can be, relative to the equity premium. As a second approach, in Section 4.2, we derive simple expressions for the equity premium and the marginal cost as functions of three elements: the dividend/price ratio, the real yield, and the expected growth rate of the economy. We also present a first estimate of the benefits of eliminating all uncertainty in U.S. corporate dividends, where we find very high costs.

\subsection{Decomposition into the fundamental risk components}

Recall that in (2.4) we have defined $V_{0}[\{X\}]$ as the time-zero implicit price of an asset that pays dividends $\{X\}$. Now define $V_{0}\left[X_{t}\right]$ as the time-zero price of an asset that pays a single dividend $X_{t}$ at time $t$. In this section, we also consider these prices at times different from zero; for instance $V_{1}\left[X_{t}\right]$ denotes the price at time $t=1$ of a security that pays $X_{t}$ at time $t$. By a no-arbitrage argument, 
the price of a consumption-equity claim equals the value of a portfolio of claims to a single dividend equal to the consumption in each period, which we call, by analogy to the terms used for bonds, strips. Thus,

$$
V_{0}\left[\{C\}_{t=1}^{\infty}\right]=\sum_{t=1}^{\infty} V_{0}\left[C_{t}\right]
$$

To use the same notation for the prices of bonds, we denote by $1_{t}$ a dividend that is equal to one at $t$ for all $z^{t} \in Z^{t}$ and zero otherwise. Define as $R_{0, t}\left[X_{t}\right]$ the time-zero return until maturity of an asset with a single payment $X_{t}$ at $t$; that is,

$$
R_{0, t}\left[X_{t}\right] \equiv \frac{V_{t}\left[X_{t}\right]}{V_{0}\left[X_{t}\right]}=\frac{X_{t}}{V_{0}\left[X_{t}\right]}
$$

Specializing the previous definition, denote the one-period holding return of an asset with single payment $X_{t}$ at $t$ as

$$
R_{0,1}\left[X_{t}\right] \equiv \frac{V_{1}\left[X_{t}\right]}{V_{0}\left[X_{t}\right]}
$$

Using these definitions, denote by $\nu_{0}$ the multiplicative (conditional) equity premium,

$$
1+\nu_{0} \equiv \frac{E_{0}\left(R_{0,1}\left[\{C\}_{t=1}^{\infty}\right]\right)}{R_{0,1}\left[1_{1}\right]}
$$

Proposition 4.1. Define the weights, $w_{0}\left[C_{t}\right]$, as follows

$$
w_{0}\left[C_{t}\right] \equiv \frac{V_{0}\left[C_{t}\right]}{V_{0}\left[\{C\}_{t=1}^{\infty}\right]}
$$

Then the multiplicative consumption-equity premium can be written as

$$
1+\nu_{0}=\sum_{t=1}^{\infty} w_{0}\left[C_{t}\right]\left(\frac{E_{0}\left(R_{0,1}\left[C_{t}\right]\right)}{R_{0,1}\left[1_{1}\right]}\right)
$$

and the marginal cost of consumption uncertainty can be written as

$$
1+\omega_{0}^{m}=\sum_{t=1}^{\infty} w_{0}\left[C_{t}\right]\left(\frac{E_{0}\left(R_{0, t}\left[C_{t}\right]\right)}{R_{0, t}\left[1_{t}\right]}\right)
$$


Equation (4.3) says that the marginal cost of consumption uncertainty equals a weighted sum of dividend strip return premia with holding period until maturity. In contrast, the multiplicative equity premium to a share that pays aggregate consumption, is equal to a weighted sum of dividend strip premia for one-period holding returns. Note that the weights, $w_{0}\left[C_{t}\right]$, are indeed the same for the marginal cost and for the equity premium.

When we compare the two expressions, (4.2) and (4.3), for the equity premium and the marginal cost, respectively, another fundamental difference becomes apparent. If the payouts are not random, then the holding returns until maturity, that determine the marginal cost, are not random either; that is,

$$
R_{0, t}\left[X_{t}\right]=R_{0, t}\left[1_{t}\right] \equiv \frac{1_{t}}{V_{0}\left[1_{t}\right]} .
$$

Thus, the multiplicative strip premium is zero,

$$
E_{0}\left(R_{0, t}\left[1_{t}\right]\right)=\frac{E_{0}\left(R_{0, t}\left[1_{t}\right]\right)}{R_{0, t}\left[1_{t}\right]}=1 .
$$

Therefore, the marginal cost is pure compensation for payout risk. This is in contrast to the one-period holding returns that make up the equity premium. Realized returns depend on the valuation at time 1 ,

$$
R_{0,1}\left[X_{t}\right]=R_{0,1}\left[1_{t}\right] \equiv \frac{V_{1}\left[1_{t}\right]}{V_{0}\left[1_{t}\right]},
$$

so that premia are in general nonzero:

$$
E_{0}\left(R_{0,1}\left[1_{t}\right]\right)=\frac{E_{0}\left(R_{0,1}\left[1_{t}\right]\right)}{R_{0,1}\left[1_{1}\right]} \neq 1 .
$$

This comparison shows that the difference between the equity premium and the marginal cost will be a function of the slope of the term structure, as we discuss in more detail for the log-linear case that follows.

\subsubsection{Log-linear environment}

We introduce a log-linear environment for two reasons. First, this environment allows us to sharpen the comparison just made between the equity premium and the marginal cost. In particular, it helps to explain the different impact of the 
term structure risk and the payout uncertainty risk on the equity premium and the marginal cost. While the distinction between term structure risk and payout uncertainty risk is a general concept, in the log-linear environment, these risk premia can be expressed in a separable way. ${ }^{7}$ Additionally, we will use the loglinear environment later to estimate the marginal cost of business cycles.

As is well known, if there are no arbitrage opportunities, then under technical regularity conditions, there must exists a non-negative process $\beta^{t} M_{t}$ such that all prices satisfy

$$
V_{0}[\{X\}]=\sum_{t=1}^{\infty} \beta^{t} E_{0}\left[\frac{M_{t}}{M_{0}} X_{t}\right]
$$

We call the process $\beta^{t} M_{t}$ a stochastic pricing kernel. We assume that the logarithm of the kernel and the logarithm of the dividends of the assets of interest can be represented as linear functions of a linear VAR model. Specifically, let $s_{t}$ be a state vector following a multivariate, homoscedastic VAR

$$
s_{t}=A s_{t-1}+\varepsilon_{t}
$$

where $\varepsilon_{t}$ is a multivariate normal vector, i.i.d. through time. The square matrix $A$ determines the dynamics of the system. We require the roots of $A$ to be all smaller than or equal to one, thus allowing for $I(1)$ nonstationarity. Assume that

$$
\begin{aligned}
\ln \left(C_{t}\right) & =\ln (1+g)^{t}+l_{c} \cdot s_{t}, \\
\ln \left(M_{t}\right) & =l_{m} \cdot s_{t},
\end{aligned}
$$

where the loadings, $l_{c}$ and $l_{m}$, are row vectors and $g$ is the trend growth rate.

Following the calculations in Jermann (1998), we can separate the multiplicative premium for one-period holding returns of a risky strip into two parts. One part is the term premium, that is, the excess return of a long bond over the short rate, and the other is a compensation for the riskiness of the payout. Thus, the equity premium can be written as

$$
1+\nu_{0}\left(s_{0}\right)=\sum_{t=1}^{\infty} w\left[C_{t}\right]\left(s_{0}\right)\left\{\frac{E_{0}\left(R_{0,1}\left[1_{t}\right]\right)}{R_{0,1}\left[1_{1}\right]} \exp \left(-\operatorname{cov} 0\left(\log M_{1}, E_{1} \log C_{t}\right)\right)\right\}
$$

\footnotetext{
${ }^{7}$ For studies that focus on the distinctions between these two types of risk, see Campbell (1986), Jermann (1998), and Abel (1999) .
} 
where we use the notation $\nu_{0}\left(s_{0}\right)$ and $w\left[C_{t}\right]\left(s_{0}\right)$ to indicate that the equity premium and the weights depend on the current state vector $s_{0}$. Notice that the equity premium is a weighted average of two terms. The first term is the expected return of a $t$-period real zero-coupon bond over the short rate. This term is higher, when the real term structure is steeper. The second term, $-\operatorname{cov} 0\left(\log M_{1}, E_{1} \log C_{t}\right)$, measures how undiversifiable is the risk about news on period $t$ consumption. ${ }^{8}$

By similar calculations the marginal cost equals

$$
1+\omega_{0}^{m}\left(s_{0}\right)=\sum_{t=1}^{\infty} w_{0}\left[C_{t}\right]\left(s_{0}\right) \exp \left(-\operatorname{cov}_{0}\left(\log M_{t}, \log C_{t}\right)\right)
$$

where $-\operatorname{cov}_{0}\left(\log M_{t}, \log C_{t}\right)$ measures how undiversifiable consumption risk at horizon $t$ is. Notice that for the marginal cost of consumption uncertainty, there is no term involving the real term structure.

In the case where $\log M_{t}$ and $\log C_{t}$ are very persistent, this covariance can be much larger in absolute value than the covariance in the expression for the equity premium.

Let us compare the covariances in the expressions for the marginal cost (4.5) and the equity premium (4.6). By definition of the coefficient of correlation, $\rho_{0}(\cdot)$,

$$
\begin{aligned}
\operatorname{cov}_{0}\left(\log M_{t}, \log C_{t}\right) & =\rho_{0}\left(\log M_{t}, \log C_{t}\right) \sigma_{0}\left(\log M_{t}\right) \sigma_{0}\left(\log C_{t}\right), \\
\operatorname{cov}_{0}\left(\log M_{1}, E_{1} \log C_{t}\right) & =\rho_{0}\left(\log M_{1}, E_{1} \log C_{t}\right) \sigma_{0}\left(\log M_{1}\right) \sigma_{0}\left(E_{1} \log C_{t}\right) .
\end{aligned}
$$

The more persistent are the processes for $\log M_{t}, \log C_{t}$, the bigger are the differences $\sigma_{0}\left(\log M_{t}\right)-\sigma_{0}\left(\log M_{1}\right)$ and $\sigma_{0}\left(\log C_{t}\right)-\sigma_{0}\left(E_{1} \log C_{t}\right)$. Thus, the more persistent these processes are, the larger the marginal cost is, relative to the equity premium. For instance, if both $\log M_{t}$ and $\log C_{t}$ are $I(1)$, then the standard deviations in the marginal cost, $\sigma_{0}\left(\log M_{t}\right)$ and $\sigma_{0}\left(\log C_{t}\right)$, grow without bound, while the standard deviations for the equity premium, $\sigma_{0}\left(\log M_{1}\right)$ and $\sigma_{0}\left(E_{1} \log C_{t}\right)$, are bounded.

Example: Separable CRRA utility. We illustrate the difference between the marginal cost of uncertainty and the equity premium using three specifications of a well-understood economy. With time-separable expected discounted CRRA utility, the kernel satisfies $\ln \left(M_{t} / M_{0}\right)=-\gamma \ln \left(C_{t} / C_{0}\right)$. The following results can be shown by direct calculations.

\footnotetext{
${ }^{8}$ Given the assumptions of the log-linear, homoscedastic system, the covariance is independent of the state $s_{0}$ and depends only on the maturity of the strip.
} 
1. Assume that consumption follows a random walk. In this case, interest rates are constant, thus there are no term premia. Payout uncertainty premia for $k$-period holdings are related to the one-period return premia by

$$
-\operatorname{cov}_{0}\left(\log M_{t}, \log C_{t}\right)=-t \operatorname{cov}_{0}\left(\log M_{1}, E_{1} \log C_{t}\right)=t \gamma \sigma_{\varepsilon}^{2}
$$

Clearly, in this case, the marginal cost, $t \gamma \sigma_{\varepsilon}^{2}$, is bigger-likely substantially so-than the consumption-equity premium, $\gamma \sigma_{\varepsilon}^{2}$.

2. Assume that consumption growth rates follow an $\mathrm{AR}(1)$ with positive serial correlation. It can then easily be shown that the term structure is downwardsloping, so that

$$
\frac{E_{0}\left(R_{0,1}\left[1_{t}\right]\right)}{R_{0,1}\left[1_{1}\right]}<1
$$

and

$$
-\operatorname{cov}_{0}\left(\log M_{t}, \log C_{t}\right)>-t \operatorname{cov}_{0}\left(\log M_{1}, E_{1} \log C_{t}\right)>0
$$

so that there are even more reasons for which the marginal cost is bigger than the consumption-equity premium.

3. The one case where the consumption-equity premium might actually be bigger than the cost of consumption uncertainty is with an $\operatorname{AR}(1)$ in consumption levels, with positive serial correlation; that is the growth rate of consumption is negatively autocorrelated. In this case, the term structure is upward-sloping,

$$
\frac{E_{0}\left(R_{0,1}\left[1_{t}\right]\right)}{R_{0,1}\left(1_{1}\right)}>1
$$

and

$$
0<-\operatorname{cov}_{0}\left(\log M_{t}, \log C_{t}\right)<-t \operatorname{cov}_{0}\left(\log M_{1}, E_{1} \log C_{t}\right)
$$

\subsection{Decomposition into yields and growth rates}

As shown in equation (2.4), the marginal cost of consumption uncertainty, can, by definition, be viewed as the ratio of the prices of two securities. For the particular case of comparing actual consumption to its expected growth path these two securities have intuitive interpretation and close real-world counterparts. Indeed, the appropriate version of equation (2.4) is

$$
1+\omega_{0}^{m}=\frac{V_{0}\left(\left\{E_{0}(C)\right\}\right)}{V_{0}(\{C\})}
$$


Here, the numerator $V_{0}\left(\left\{E_{0}(C)\right\}\right)$ is a perpetual bond with coupons that are growing at the expected consumption growth rate, and the denominator, $V_{0}(\{C\})$ is a consumption-equity claim. Dividing both the numerator and the denominator of the fraction by $C_{0}$, we have,

$$
1+\omega_{0}^{m}=\frac{V_{0}\left(\left\{E_{0}(C)\right\}\right) / C_{0}}{V_{0}(\{C\}) / C_{0}},
$$

which suggests that the marginal cost is a function of two price/dividend ratios, or reciprocally, two yields. If we assume that aggregate consumption has a constant expected trend growth rate, $g_{0}$, this can be written as

$$
1+\omega_{0}^{m}=\frac{\sum_{i=1}^{\infty}\left(\frac{1+g_{0}}{1+r_{0}}\right)^{i}}{\frac{1}{d p_{0}}}=\left(1+g_{0}\right)\left[\frac{d p_{0}}{r_{0}-g_{0}}\right],
$$

where $r_{0}$ is the real yield of a bond with the duration of the growing perpetuity, and $d p_{0}$ is the dividend yield of the consumption-equity.

In general, the expected excess return of equity cannot be written as a function of $r_{0}, d p_{0}$, and $g_{0}$. Nevertheless, if we make some simplifying assumptions, we obtain an expression that depends on similar factors. If we assume that dividend yields are constant, then we can write the expected return of equity as $\left(1+g_{0}\right)$. $\left(1+d p_{0}\right)$. By definition, the equity premium is the expected excess return of equity over a riskfree investment yielding a known return of $y_{0}$. Thus,

$$
1+\nu_{0}=\frac{\left(1+g_{0}\right) \cdot\left(1+d p_{0}\right)}{\left(1+y_{0}\right)} .
$$

In general, $y_{0}$ will be smaller than the yield of the long-term bond $r_{0}$ used in equation (4.7). These yields will be equal only in special cases, for instance, if the term structure is flat. Clearly, the formula for the equity premium in (4.8) is different from the one for the marginal cost in (4.7). Notice that the equity premium $\nu_{0}$ in (4.8) is approximately $\nu_{0} \simeq g_{0}+d p_{0}-y_{0}$. Hence, it cannot exceed a few percentage points. This is quite different from the marginal cost, $\omega_{0}^{m}$, in equation (4.7), which is approximately $\omega_{0} \simeq d p_{0} /\left(r_{0}-g_{0}\right)$ and hence could be much larger. Below we provide quantitative content to equation (4.7) by measuring the marginal cost. 


\subsubsection{A measure of the marginal cost of dividend uncertainty}

For a first quantitative cut, we plug historical averages into the formula for the marginal cost given by equation (4.7). Indeed, we can consider the dividend/price ratio, $d p$, to be the historical dividend/price ratio of the value-weighted U.S. stock market. Of course, we are thus looking at how dividend uncertainty reduces the price of equity relative to a perpetual bond paying as coupons the expected dividends; we will focus directly on consumption fluctuations in the Section $5 .^{9}$

Combining the average historical dividend/price ratio with a historical longterm real yield and the dividend growth rate, we report in Table 1 a marginal cost for dividend uncertainty of $619 \%$ and $174 \%$ for the periods $1889-1997$ and 1954-97, respectively. That is, for the long sample period, the buyers of an equity claim would have to be given a more than seven times higher dividend to make them value equity as much as a perpetual bond paying coupons growing at the $1.31 \%$ trend growth rate of dividends. Notice that the lower cost of dividend uncertainty for the postwar period corresponds, indeed, to a period of lower volatility as measured by the standard deviations of the growth rates of consumption and dividends. If dividends were equal to aggregate consumption, we would have had here our estimate of the marginal cost of consumption uncertainty. The additional statistics in Table 1 document some aspects of dividend and consumption behavior.

\section{Measuring the Marginal Cost of Business Cycles}

The approach used to measure the cost of business cycles in Section 4.2.1 has the advantage of being simple and intuitive, but it has several shortcomings. First, it measures the elimination of all the uncertainty, as opposed to just that of business cycle fluctuations. Second, the theory requires the use of an asset which has dividends that are perfectly correlated with consumption. The measurement in Section 4.2.1 uses a diversified equity portfolio, which has dividends that display positive correlation with consumption, but a correlation much smaller than one. Third, the calculation of the marginal cost can be very sensitive to small inconsistencies in the measurement of the different yields and growth rates. This is due to

\footnotetext{
${ }^{9}$ To be precise, the expected marginal cost should be estimated by the average of the corresponding marginal costs. Here we compute the marginal cost for the average yields, average dividend/price ratios, and average growth rates, since we only observe ex-post real yields and growth rates.
} 
the nonlinearities in (4.7), where $d p_{0}$ is divided by $r_{0}-g_{0}$. For instance, the calculation of the duration-adjusted yield, $r_{0}$, is not straightforward without specifying jointly the term structure and the expected growth rate of consumption.

In this section, we address these three shortcomings. First, we isolate the cost of consumption fluctuations corresponding to business cycle frequencies and compare it to the cost of all consumption uncertainty that is usually computed in the literature. Specifically, we isolate cyclical consumption movements corresponding to cycles of at most 8 years by using a band-pass filter approach. Second, we price an asset that pays aggregate consumption as opposed to aggregate dividends. Third, by explicitly modeling a pricing kernel, we make our estimates of dividend yields and growth rates consistent with each other. As a way to check our quantitative results, and to provide intuition, we also examine the special case where the logarithms of consumption and the pricing kernel follow random walks. We derive a simple expression for the marginal cost of consumption fluctuations, and we provide an estimate with a pricing kernel that is required to fit the excess returns of different CRSP portfolios.

Let us start here by overviewing the steps involved in our estimation procedure. A more detailed step-by-step discussion follows. Based on the log-linear environment defined in Section 4, we

1. Select state variables that determine marginal utility of consumption based on popular models and estimate a linear autoregressive law of motion for the logarithm of these state variables, $s_{t}$.

2. Estimate the loading vector for the pricing kernel, $l_{m}$, and the effective time discount rate, $\beta$, from a set of asset pricing moment conditions determined by the theoretical considerations discussed above.

3. Specify the trend $\left\{\mathfrak{C}_{\tau}\right\}_{t=\tau}^{\infty}$, compute $V_{t}\left(\left\{\mathfrak{C}_{\tau}\right\}_{\tau=1}^{\infty}\right)\left(s_{t}\right)$ and $V_{t}\left(\left\{C_{\tau}\right\}_{\tau=1}^{\infty}\right)\left(s_{t}\right)$ for every $s_{t}$ of our dataset, and report the sample mean of the marginal costs.

\subsection{The state variables}

We choose a multidimensional state vector to capture the dependence of marginal utility on noncontemporaneous consumption. This dependence has been emphasized in the literature in models with habit formation and in models with non-expected utility. 
The state variables include (1) aggregate consumption, (2) aggregate dividends, (3) the dividend/price ratio, (4) the long-short government bond yield spread, and (5) the value-weighted realized stock return. Our choice of these variables is motivated by empirical work on intertemporal asset pricing. For instance, Campbell (1996) has used dividend/price ratios, yield spreads, realized stock returns, and aggregate output as his state variables. We include consumption in the state space since we need to estimate the price of an asset with payouts equal to consumption.

We use annual time series covering the period 1889-1997. Data sources and definitions are described in detail in Appendix A. We start by removing a linear deterministic growth trend from consumption and dividends. Specifically, we extract a drift that is computed as the mean of the $(\log )$ growth rates. We also demean all variables. For instance, starting with raw consumption $C_{t}$, we get $c_{t}=\log \left(C_{t}\right)-c_{0}-\mu t$, where the drift and the mean are defined, respectively, as $\mu \equiv$ mean $\left(\log \Delta c_{t}\right)$ and mean $\left(c_{t} ; c_{0}\right)=0$. We then estimate by OLS a VAR for growth rates for consumption and dividends and for levels for the remaining variables. We estimate systems with and without an error-correction term that forces cointegration between consumption and dividends. A system in levels, as in equation (4.4), is then recovered. The variance-covariance matrix of the innovations is obtained from the residuals of the estimated VAR.

By estimating the VAR in growth rates for consumption and dividends, we have introduced one or two unit roots into the matrix $A$, depending on whether we introduce an error-correction term. This decision to model consumption and the pricing kernel as nonstationary has first-order quantitative implications for the measurement of the cost of consumption uncertainty. It is certainly possible to argue on statistical grounds that consumption or dividends or both can be modeled as (trend) stationary processes. However, we select a nonstationary representation for consumption due to the fact that a stationary pricing kernel cannot possibly explain simultaneously the relatively flat historical term structure of interest rates and the relatively large historical equity premium. Intuitively, the reason is that a stationary pricing kernel that is volatile enough to generate high equity risk premia has too much predictable movements to be consistent with the relatively low term spread. This result, developed in Alvarez and Jermann (2000), applies not only to the particular specification considered here, but to any stationary pricing kernel. Given the requirement of a nonstationary pricing kernel, we choose consumption and dividends to be nonstationary. We think that specifying consumption to be stationary while having a nonstationary pricing 
kernel would be an a priori unappealing property of any representation.

\subsection{Estimating the loading vector $l_{m}$ and the time discount factor $\beta$}

Once the law of motion of the state vector is estimated, we need to estimate the various loading coefficients on the states, $l_{m}$, plus the time discount factor, $\beta$. We will choose $\left(l_{m}, \beta\right)$ in order to minimize $\Im=\left[\theta-f\left(l_{m}, \beta\right)\right]^{\prime} \cdot W \cdot\left[\theta-f\left(l_{m}, \beta\right)\right]$, where $\theta$ is a vector of moments to match, $f\left(l_{m}, \beta\right)$ contains the corresponding moments generated by our asset pricing model, and $W$ is a weighting matrix.

Our analysis in Section 4 of the fundamental components of the equity premium and of the marginal cost of consumption uncertainty suggests that we should focus on a pricing kernel that is good at explaining historical aggregate stock price behavior and the real term structure. Specifically, we choose the asset pricing kernel to replicate the U.S. average dividend/price ratio and the U.S. equity premium. We consider two ways of replicating the equity premium. First, by applying the pricing kernel to the estimated process of dividends, our model generates a series of conditional equity premia for which we compute the sample mean

$$
f_{j}\left(l_{m}, \beta\right)=\frac{1}{T} \sum_{t=1}^{T}\left[1+\nu_{t}^{d}\left(s_{t} ; l_{m}, \beta\right)\right]
$$

where $\nu_{t}^{d}(\cdot)$ is defined as $\nu(\cdot)$ in equation (4.5) except that here the payout process represents aggregate dividends instead of aggregate consumption. Our second moment condition relative to the equity premium applies the pricing kernel to realized U.S. excess stock returns, $V W R_{t, t+1}-R_{t, t+1}^{f}$, so that

$$
f_{i}\left(l_{m}, \beta\right)=\frac{1}{T} \sum_{t=1}^{T} \beta \exp \left(l_{m}\left(s_{t+1}-s_{t}\right)\right) \cdot\left(V W R_{t, t+1}-R_{t, t+1}^{f}\right),
$$

with $\theta_{i}=0$. In Table 2, we refer to these two conditions as $E\left(R^{d} / R^{f}\right)$ and $E\left(V W R-R^{f}\right)$, respectively. We also make the kernel fit the average real return on a riskfree short-term bond and the average real yield on a 20-year bond. ${ }^{10}$

\footnotetext{
${ }^{10}$ Practically, we make the model fit the average yield of a zero-coupon bond of 13 years maturity, given that this corresponds approximately to the duration of the 20-year government coupon bond in our historical data set.
} 


\subsection{Defining business cycles}

We now describe how we specify the process corresponding to the consumption trend $\{\mathfrak{C}\}$. We choose two approaches. As our first approach, we specify the logarithm of the consumption trend to be a one-sided moving-average of current and past consumption, $\ln \left(\mathfrak{c}_{\tau}\right)=\sum_{k=0}^{K} a_{k} \cdot c_{\tau-k}$, for appropriate moving average coefficients. ${ }^{11}$ As our second approach, we set trend consumption equal to its conditional expectation, $\mathfrak{C}_{\tau}=E_{t}\left(C_{\tau}\right)$.

We define business cycles to be the cyclical movements that last 8 years or less, so that our trend $\{\mathfrak{C}\}$ contains movements of more than 8 years. Assuming business cycles to last up to 8 years is consistent with the definition of Burns and Mitchell (1946) and with many recent studies describing business cycle properties. This also corresponds approximately to the definition of business cycles implied by the widely used Hodrick-Prescott filter for quarterly data with a smoothing parameter of $1600 .{ }^{12}$ For comparison, we also report results for trends with frequency cutoffs at 12,16 , and 20 years.

We choose the moving-average coefficients $\left\{a_{k}\right\}$ so as to represent a bandpass filter that lets pass frequencies that correspond to cycles of $Y$ years and more. Band-pass filters are represented in the time domain by infinite-order twosided moving averages. However, a requirement of our analysis is to have trend consumption in time $t$ be function of information available at time $t$, thus, our choice of a one-sided moving average.

We choose the moving-average coefficients of our trend consumption using the procedures presented by Baxter and King $(1998,1999)$. Let $\beta(\omega)$ be the frequency response function of the desired low-pass filter, which in our case is equal to one for frequencies lower than $Y$ years and zero otherwise. Let $\alpha_{K}(\omega)$ be the frequency response function associated with a set of moving-average coefficients $\left\{a_{k}\right\}_{k=1}^{K}$. We select the moving-average coefficients $\left\{a_{k}\right\}$ so that $\alpha_{K}$ approximates $\beta$. In particular, our choice of $\left\{a_{k}\right\}$ minimizes

$$
\int_{\pi}^{-\pi}\left|\beta(\omega)-\alpha_{K}(\omega)\right|^{2} f(\omega) d \omega
$$

where $f(\omega)$ is a weighting function representing (an approximation to) the spectral density of the series to be filtered. In this minimization, we impose the condition $\alpha_{K}(0)=1$, which implies that $\sum_{k=0}^{K} a_{k}=1$.

\footnotetext{
${ }^{11}$ With this definition of the trend $\mathfrak{C}, E_{0}\left(C_{t}\right) \neq E_{0}\left(\mathfrak{C}_{t}\right)$, because $\mathfrak{C}$ is a nonlinear function of $C$. Quantitatively, however, this discrepency is negligible, as we show in Appendix B.

${ }^{12}$ For a detailed analysis, see Baxter and King (1999).
} 
We fix the number of lagging moving-average coefficients at fifteen (that is, $K=15)$, and we use the spectral density of an AR(1) with autocorrelation 0.99 for the weighting function $f$, because this matches approximately the spectral density of consumption.

Unfortunately, one-sided filters are far from perfect low-pass filters. Being one-sided, these filters cannot avoid introducing a phase shift; that is, the trend and its associated deviations will be lagging the series. The objective function displayed above is closely related to the variance of the difference of the desired series and the implied filtered series. Thus, this objective function trades-off the phase shift of the filtered series with the desired shape of the spectral density. ${ }^{13}$ This can be seen by plotting the transfer function of the filter as in Figure 2 in the left panel. The transfer function should be one in-between the desired frequencies and zero for higher frequencies. Instead, it tends to let pass up to $30 \%$ of the variance at higher frequencies, so that the computed trend contains a substantial amount of cyclical variability. As shown in the right panel of Figure 2 , the corresponding two-sided band-pass filter fits the ideal filter's step function much closer-remember that a symmetric two-sided filter does not introduce a phase shift. The corresponding time-domain representation is in Figure 3A in the first panel. Clearly, the one-sided filter generates cyclical movements that are less volatile than those from the corresponding two-sided filter shown by the thin line. The second panel in Figure 3A and both panels in Figure 3B present consumption deviations from trends corresponding to cutoffs of 12,16 , and 20 years, allowing for comparisons with the deviations obtained with the two-sided filter for 8-year cycles.

Based on these comparisons, we decide to make an ad hoc adjustment to the one-sided filter so as to replicate the amount of business cycle volatility obtained

${ }^{13}$ This can be seen by rewriting the objective function as follows:

$$
\begin{array}{rl}
\int_{-\pi}^{\pi}\left|\beta(\omega)-\alpha_{K}(\omega)\right|^{2} & f(\omega) d \omega=\int_{-\pi}^{\pi}\left(|\beta(\omega)|-\left|\alpha_{K}(\omega)\right|\right)^{2} f(\omega) d \omega \\
& +\int_{-\pi}^{\pi} 2 \beta(\omega)\left|\alpha_{K}(\omega)\right|\left[1-\cos \left(\psi_{K}(\omega)\right)\right] f(\omega) d \omega
\end{array}
$$

where $\psi_{K}(\omega)$ denotes the phase shift of the filter. Thus, the objective function can be written as the square of the differences of the gains of the filters, $\left(|\beta(\omega)|-\left|\alpha_{K}(\omega)\right|\right)^{2}$, plus a term that depends on the phase shift. This second term is zero, if the filter has no phase shift. For instance, a symmetric two-sided filter has no phase shift, so it also fits the gain of the approximate filter (and thus the transfer function). Instead, a one-sided filter has to find the trade-off between these two forces. 
from the more accurate two-sided filter. As shown in Figure 3A, the series generated by the one-sided filter is strongly correlated with the series from the two-sided filter, but the series generated by the one-sided filter is less volatile. Thus, we scale up the volatility by multiplying the cyclical deviations by a constant $\theta>1$. This is achieved by defining the adjusted trend, $\mathfrak{c}_{t}^{*}$, as

$$
\mathfrak{c}_{t}^{*}=(1-\theta) c_{t}+\theta \mathfrak{c}_{t} .
$$

With $\theta=1.4$, the standard deviation of the scaled one-sided filter is about equal to the one from the two-sided filter. The implied transfer function of the adjusted low-pass filter defining the trend is presented in Figure 4: the deviations from the ideal filter are somewhat larger around the cutoff frequencies; for the business cycle frequencies, the adjusted filter represents a significant improvement. As we show below, the approximate marginal cost implied by the adjusted filter is obtained by the same scaling, so that $\omega^{m *} \cong \theta \cdot \omega^{m}$.

We also report results based on some other popular moving-average filters. A geometric filter is specified so that $a_{k}=\delta_{g}^{k+1}$ and $\sum_{k=0}^{K} a_{k}=1$, for $K=5$; thus, $\delta_{g}=0.5041$. A linear filter is specified so that $a_{k}=\delta_{l}\left(1-\frac{k}{K+1}\right)$ and $\sum_{k=0}^{K} a_{k}=1$, which gives a slope so that the next potential weight $a_{K+1}=0$; thus, $\delta_{l}=0.2857$ with $K=5$. We choose a lag length of $K=5$ because the one-sided frequency domain filters with 15 lags that we use have moving-average coefficients that are not too different from zero for lags larger than 5. Figure 3C shows the cyclical consumption components in these cases.

\subsection{Findings and discussion}

In Tables 2A-2D, we report estimates for various state-space systems, various loading states, and various moments to fit for the complete sample and for the postwar period. We have chosen the following algorithm for minimizing the sum of squares of the discrepancies between model-generated moments and the data, $\Im$. We start by estimating systems with as many coefficients as there are moments, using a diagonal weighting matrix that assigns a weight of $1 / 5$ to the moments involving the equity premium and 1 for the remaining moments. As shown in Tables 2A-2D in the last column entitled 'Fit,' for many systems we are able to drive $\Im$ to 0 . As a minimum requirement for fit, we impose a maximum discrepancy of $1.5 \%$ for the moments involving the equity premium and $0.3 \%$ for the others. In some cases, we are able to obtain a satisfactory fit only after removing one of the forecasting variables or changing slightly the sample period. 
If this exactly identified approach fails to provide a satisfactory fit, we move to an overidentified system marked by 'OI,' by adding one moment condition, and we report the result with the lowest $\Im$.

Overall, for fluctuations defined by the 8-year filter, we find on average a marginal cost of about $0.40 \%$ of lifetime consumption for the period 1889-1997 and of about $0.20 \%$ for the period 1954-97. With our adjustment to the one-sided filter, as explained in the previous section, with $\theta=1.4$, we estimate the marginal cost of business cycles to be about $0.55 \%$ based on the entire sample and about $0.30 \%$ for the postwar period. Imposing cointegration between consumption and dividends yields an estimate of a few basis points higher than without cointegration. ${ }^{14}$ For comparison, the linear and the geometric filters imply marginal costs of about $0.75 \%$ and $1.75 \%$ of consumption for the entire sample period and the postwar period, respectively.

The column labeled 'inf,' reports the cost of all consumption uncertainty, that is, including cycles of any length. Specifically, $\mathfrak{C}_{\tau}=E_{t}\left(C_{\tau}\right)$. We find very large costs. With cointegrated consumption and dividends, many systems yield costs at around $30 \%$ of lifetime consumption; without cointegration, they amount in general to several hundred percent. These numbers are consistent with the orders of magnitudes we found for the cost of dividend uncertainty in Section (4.2.1). These numbers are also consistent with the intuition provided by the log-linear random walk case in section (4.1.1). Fundamentally, consumers very much dislike the possibility that consumption can wander off very far from its expected path - consistent with this interpretation, the additional structure provided by the cointegration restriction yields lower costs. ${ }^{15}$

Across the different specifications, our measures are robust for the different moving-average filters, even though the loading coefficients on some states differ, sometimes substantially, across cases. There is more variability for measures of consumption uncertainty. One reason for the variability in measuring the costs of consumption uncertainty is that in some cases prices for the perpetual bonds with growing coupons become very large. That is, long-term yields are low relative to the trend growth rate.

\footnotetext{
${ }^{14}$ Taking an average over all reported estimates corresponding to the 8-year filter in Table 2 , we have $0.35 \%$ and $0.42 \%$ for the period $1889-1997$ without and with cointegration between consumption and dividends, $0.17 \%$ and $0.22 \%$ for the period $1954-97$ without and with cointegration.

${ }^{15}$ As explained in Section 5.2 we model consumption as nonstationary because a stationary consumption process is not consistent with having a kernel that can simultaneously explain a large equity risk premium and a relatively flat real term structure.
} 


\subsection{The marginal cost of fluctuations in a simpler case}

In this section, we specialize the kernel and the payoffs by assuming that they are given by random walks. Under the random walk assumption, most of the dynamics are eliminated: interest rates, dividend/price ratios, and expected excess returns are all constant. For this reason, we can present the marginal cost in a simple and intuitive form. The marginal cost of fluctuations is approximately equal to the consumption-equity premium multiplied by a simple expression of $\left\{a_{i}\right\}$, the moving-average coefficients that define $\left\{\mathfrak{C}_{t}\right\}$ as a function of $\left\{C_{t}\right\}$. Specifically, for the 8-, 12-, 16- and 20-year filters the marginal cost of fluctuations equals approximately $0.38,0.72,0.77$, and 0.88 times the consumption-equity premium. We show that the consumption-equity premium equals the covariance between the pricing kernel and the consumption growth rate, and we estimate this covariance by fitting a pricing kernel to average excess returns. We find that the results are similar to those obtained fitting a more general version of the pricing kernel in Section 5.4. Additionally, we explore the consequences of introducing more assets, namely, CRSP size-decile portfolios, into our analysis.

We start by specializing the log-linear framework to the following:

$$
s_{t+1}=s_{t}+\varepsilon_{t+1},
$$

where $\varepsilon_{t+1}$ is multivariate normal i.i.d through time with mean zero. In this case, the pricing kernel can be written as

$$
\Delta m_{t+1} \equiv \log \frac{M_{t+1}}{M_{t}}=l_{m} \cdot \varepsilon_{t+1} .
$$

Notice that implicit in this specification of the state space is the assumption that consumption and dividends of any asset included in the state space follow random walks, possibly with correlated innovations. Let $D_{t}$ be the dividend of any such

asset, and let $\Delta d_{t+1} \equiv \log \frac{D_{t+1}}{D_{t}}$. Under these assumptions, we have the following well-known results.

Proposition 5.1. If the $\log$ pricing kernel and the $\log$ dividends are random walks with homoscedastic innovations, then interest rates, dividend/price ratios, and expected returns of equities are all constant; moreover, the expected returns of strips are all equal to the expected returns of the corresponding equity claims. Furthermore, if the innovations are normal, then the ratio of the expected return of a risky strip paying $D_{t+s}$ in period $t+s$ to the one-period interest rate $R_{f}$ and 
the excess return for equity paying dividends equal to the process $\{D\}$ are given by

$$
\frac{E_{t}\left(R_{t+1}[\{D\}]\right)}{R_{f}}=\frac{E_{t}\left(R_{t+1}\left[D_{t+s}\right]\right)}{R_{f}}=\exp \left(-\operatorname{cov}\left(m_{t+1}, d_{t+1}\right)\right) \text { for all } s \geq 1 .
$$

If $E\left(R_{t+1}^{e}\right)$ is a vector of expected excess returns, then the loading vector is given by

$$
\log E\left(R_{t+1}^{e}\right)=l_{m} \cdot \Sigma
$$

where $\Sigma=\left[\operatorname{cov}\left(\Delta s_{t+1, i}, \Delta d_{t+1, j}\right)\right]_{i, j}$ is the covariance matrix of the innovations in the growth rate of state $i$ with the innovations in the growth rate of the dividends of stock $j$.

Under the assumptions used in the Proposition 5.1, dividend growth rates and total returns are perfectly correlated, since dividend/price ratios are constant. In our implementation, we use consumption or returns of broad indices as states. With consumption as the dividend, Proposition 5.1 says that the multiplicative excess return of consumption-equity equals the negative covariance of the kernel with consumption growth:

$$
\frac{E\left(R_{t+1}\left[\left\{C_{t+s}\right\}_{s=1}^{\infty}\right]\right)}{R_{f}}=\exp \left(-\operatorname{cov}\left(\Delta m_{t+1}, \Delta c_{t+1}\right)\right) .
$$

Now we present a proposition that links the multiplicative excess return of consumption-equity to the moving-average coefficients of the trend $\left\{\mathfrak{C}_{t}\right\}$.

Proposition 5.2. Let the trend $\mathfrak{C}_{t}$ be defined as

$$
\log \mathfrak{C}_{t}=\tau(t)+\sum_{i=0}^{K} a_{i} \log C_{t-i}
$$

where $\tau(t)$ are constant chosen so that

$$
E_{0}\left(\mathfrak{C}_{t}\right)=E_{0}\left(C_{t}\right) .
$$

Under the assumption of the log-linear system the ratio between the price of a risky strip paying $\mathfrak{C}_{t}$ and a strip paying $C_{t}$ is given by

$$
\frac{V_{0}\left[\mathfrak{C}_{t}\right]}{V_{0}\left[C_{t}\right]}=\exp \left[\operatorname{cov}_{0}\left(m_{t}, \sum_{j=0}^{J} a_{j} c_{t-j}\right)-\operatorname{cov}_{0}\left(m_{t}, c_{t}\right)\right] .
$$


Furthermore, if the pricing kernel and consumption are random walks then for the strips for which $t>K$,

$$
\frac{V_{0}\left[\mathfrak{C}_{t}\right]}{V_{0}\left[C_{t}\right]}=\left(\frac{E\left(R_{1}\left[C_{t}\right]\right)}{R_{f}}\right)^{\left[1-a_{0}+1-\left(a_{0}+a_{1}\right)+\cdots 1-\left(a_{0}+a_{1} \ldots+a_{K}\right)\right]} .
$$

The role of the constants $\tau(t)$ is to ensure that $\mathfrak{C}_{t}$ has the same conditional expectation as $C_{t}$. This adjustment involves a Jensen's inequality correction, due to our assumption that the logarithm of $\mathfrak{C}_{t}$, as opposed to just its level, is a moving-average of the logarithms of $C_{t}$. We show in Appendix B that this adjustment is quantitatively negligible.

These propositions complement our earlier results. Proposition 5.1 explains how we identify the consumption equity premium by using excess return on securities. Proposition 5.2 explains how we use that information and the values of the moving-average coefficients to measure the marginal cost of fluctuations. In particular, recall that the marginal cost of economic fluctuations is given by the ratio of the prices of a claim paying trend consumption relative to a claim paying consumption. The ratio of the prices of these long-lived securities can be written as

$$
\frac{V_{0}[\{\mathfrak{C}\}]}{V_{0}[\{C\}]}=\sum_{t=1}^{\infty} w_{t}\left[C_{t}\right] \frac{V_{0}\left[\mathfrak{C}_{t}\right]}{V_{0}\left[C_{t}\right]}
$$

where $w_{t}\left[C_{t}\right]=\frac{V_{0}\left[C_{t}\right]}{V_{0}[\{C\}]}$. Thus, the marginal cost of economic fluctuations is given by a weighted average of the ratio of strip prices. The ratio of these strip prices, with the exception of the strips for the first $K$ securities, is independent of the period in which they pay and equal to the expression in (5.3). Therefore this expression is also approximately equal to the marginal cost

$\omega^{m} \cong \log \left(1+\omega^{m}\right) \cong\left(r_{c e}-r_{f}\right)\left[1-a_{0}+1-\left(a_{0}+a_{1}\right)+\cdots+1-\left(a_{0}+a_{1} . .+a_{K}\right)\right]$,

where $r_{c e}-r_{f}$ stands for the difference between the net expected return of consumption equity and the net riskfree interest rate. The second approximation is due to the first few strips for which $j \leq K$. As we discuss in Appendix B, the approximation error is negligible for our applications.

Equation (5.4) has the following interpretation: the difference $r_{c e}-r_{f}$ captures the risk premium associated with consumption; the factor $\left[1-a_{0}+\cdots\right]$ captures the variability of the deviations from trend. To understand how this factor works, consider the case of moving-average coefficients $\left\{a_{i}\right\}$ that are positive and decreasing in $i$ and consider the following change: increase the values of $a_{i}$ corresponding 
to long lags (large $i$ ), and decrease those corresponding to earlier lags (small $i$ ). This operation increases the factor and thus the marginal cost. This operation also makes the trend smoother, since it distributes the weights more evenly. Consequently, the deviations from trend are more volatile, and thus a higher marginal cost makes sense.

In Table 3, we present computations for the expression (5.4) representing the marginal cost. As states, we use consumption or returns to various CRSP portfolios. The loading vector is fitted to the mean log excess returns of various CRSP return portfolios. The table also contains summary statistics, such as standard deviations and correlations of consumption growth rates and the market returns that illustrate which factors are driving the estimates.

We would like to highlight three types of findings. First, estimates of the consumption-equity premium lie between $0.22 \%$ and $1.66 \%$, well below the premium for the market return, which is above $7 \%$. The low estimate is due to the relatively low consumption volatility and the correlation properties of consumption, returns and the states. Second, results for the marginal cost of consumption fluctuations are similar to our estimates obtained from the more general specification in Section 5.4. And third, adding additional equity portfolios, such as the CRSP size-decile portfolios, does not change the results much. This is due to the fact that once a portfolio has been included, additional portfolios have small extra power to explain the variance of the consumption growth rate.

In Section 5.3, we have proposed an ad hoc adjustment for the one-sided filter that consists in scaling up the volatility of the cyclical component by the constant $\theta$. The next proposition shows how this adjustment affects the implied marginal cost.

Proposition 5.3. Let the trend $\mathfrak{C}_{t}^{*}$ be defined as

$$
\begin{aligned}
\log \mathfrak{C}_{t}^{*} & =\tau^{*}(t)+(1-\theta) \log C_{t}+\theta \log \mathfrak{C}_{t} \\
& =\tau^{*}(t)+(1-\theta) \log C_{t}+\theta \sum_{i=0}^{K} a_{i} \log C_{t-i}
\end{aligned}
$$

where the $\tau^{*}(t)$ are constant chosen so that

$$
E_{0}\left(\mathfrak{C}_{t}^{*}\right)=E_{0}\left(C_{t}\right)
$$

Under the assumption of the log-linear system, the ratio between the price of a 
risky strip paying $\mathfrak{C}_{t}$ and a strip paying $C_{t}$ is given by

$$
\frac{V_{0}\left[\mathfrak{C}_{t}\right]}{V_{0}\left[C_{t}\right]}=\theta \exp \left[\operatorname{cov}\left(m_{t}, \sum_{j=0}^{J} a_{j} c_{t-j}\right)-\operatorname{cov} v_{0}\left(m_{t}, c_{t}\right)\right] .
$$

Furthermore, if the pricing kernel and consumption are random walks then for the strips for which $t>K$,

$$
\frac{V_{0}\left[\mathfrak{C}_{t}\right]}{V_{0}\left[C_{t}\right]}=\left(\frac{E\left(R_{1}\left[C_{t}\right]\right)}{R_{f}}\right)^{\theta\left[1-a_{0}+1-\left(a_{0}+a_{1}\right)+\ldots+1-\left(a_{0}+a_{1} \ldots+a_{K}\right)\right]} .
$$

Given that the Jensen's inequality terms determining $\tau^{*}(t)$ are of second-order importance, the marginal cost with the adjusted trend is approximately equal to the original marginal cost scaled by $\theta$; that is,

$$
\omega^{* m} \cong \theta \cdot \omega^{m} \text {. }
$$

We end this section by illustrating the effect of a phase shift for the filter that defines the trend consumption. Given that we have defined the trend as a onesided moving average, we have introduced a phase shift. The effect of such a phase shift is to make the marginal cost larger. The intuition is that a phase shift makes trend consumption more desirable since it becomes closer to the expected value. To be precise, we show that if a trend - defined by moving average coefficients $\left\{a_{i}\right\}$ - is lagged by one period, then the cost of fluctuations goes up by the value of the consumption-equity premium.

Proposition 5.4. Let $a^{K}=\left\{a_{i}\right\}_{i=0}^{K}$ be the moving-average coefficients that define the consumption trend, and let $m\left(a^{K}\right)$ be the factor that multiplies the consumption-equity premium giving the corresponding marginal cost of fluctuations. Consider the moving-average coefficients $a^{K+1}$ satisfying

$$
a_{0}^{K+1}=0, \text { and } a_{i+1}^{K+1}=a_{i}^{K} \text { for } i=1,2, \ldots, K .
$$

Then $m\left(a^{K+1}\right)=m\left(a^{K}\right)+1$.

If we denote by $\omega^{m}(a)$ the marginal cost for a trend corresponding to movingaverage coefficients $a$, then Propositions 5.1 and 5.2 imply that

$$
\omega^{m}\left(a^{K+1}\right)-\omega^{m}\left(a^{K}\right) \cong r_{c e}-r_{f} .
$$

This means that with a phase shift of a year, the cost of business cycles increases by an amount equal to the consumption-equity premium. 


\section{Growth and Economic Fluctuations}

In this section, we analyze the relationship between growth and economic fluctuations. We consider this issue along two dimensions. First, we use our framework to estimate the benefit from increasing growth. We find the benefit from an increase in the annual growth rate of $1 \%$ to be substantial. It is about $50 \%$ of lifetime consumption with cointegrated dividends and consumption and even larger without cointegration. Second, we present a modified version of our framework that allows us to directly estimate the trade-off between long-term growth and fluctuations. We find that reducing business cycle fluctuations is valued at less than 1 basis point increase in the per annum long-term growth rate of consumption.

\subsection{The benefits from increasing growth}

We can use our definition of $\Omega$ to analyze the benefits of a permanent increase in the growth rate of the economy, as in Lucas (1987). For that, we simply let $\{\mathfrak{C}\}$ be defined as

$$
\mathfrak{C}_{t}\left(z^{t}\right)=[1+x]^{t} C_{t}\left(z^{t}\right)
$$

With $x>0$, this is a special case of our framework and all the results derived in Section 3 for the general case directly apply.

The marginal cost can be written as

$$
1+\Omega^{\prime}(0)=\frac{\sum_{t=1}^{\infty} V_{0}\left[(1+x)^{t} C_{t}\right]}{\sum_{t=1}^{\infty} V_{0}\left[C_{t}\right]}=\frac{\sum_{t=1}^{\infty}(1+x)^{t} V_{0}\left[C_{t}\right]}{\sum_{t=1}^{\infty} V_{0}\left[C_{t}\right]}=\sum_{t=1}^{\infty}(1+x)^{t} w_{0}\left[C_{t}\right],
$$

where the weights

$$
w_{0}\left[C_{t}\right]=\frac{V_{0}\left[C_{t}\right]}{\sum_{j=1}^{\infty} V_{0}\left[C_{j}\right]}
$$

are the same as in the equity premium and the cost of consumption uncertainty. Based on our estimated pricing kernel, Table 3 reports the (marginal) benefits of increasing the growth rate by $1 \%$, that is, for $x=0.01$. The estimates are high, about $50 \%$ of lifetime consumption with cointegrated dividends and consumption and higher without cointegration. These numbers are larger than the one computed by Lucas, which is $17 \%$.

To gain some intuition about these results, we consider again the random walk specification, where

$$
\Omega^{\prime}(0)=\sum_{t=1}^{\infty}(1+x)^{t} w_{0}\left[C_{t}\right]-1=\frac{\gamma\left(1+r_{c e}\right)}{1+r_{c e}-\left(1+g_{c}\right)(1+x)} \cong \frac{x}{r_{c e}-g_{c}-x}
$$


with $g_{c}=E\left[\frac{C_{t+1}}{C_{t}}\right]-1$ the net rate of consumption growth. ${ }^{16}$ In Section 5 , we estimated $r_{c e}-r_{f}$ to be less than $1.5 \%$. Thus if the riskfree rate is $r_{f}=2.5 \%$ and the growth rate of consumption $g_{c}=2 \%$, then $\Omega^{\prime}(0)=0.01 /(0.015+0.025-0.02-$ 0.01 ) equals $100 \%$; with a smaller consumption-equity premium, the marginal cost increases further. ${ }^{17}$

\subsection{The trade-off between fluctuations and growth}

In order to directly measure the trade-off between fluctuations and growth, we modify our framework. Instead of the cost of fluctuations being measured by the uniform compensation $\Omega$, now the compensation for fluctuations is expressed in terms of additional long-term growth. We find that eliminating business cycle fluctuations is worth less than 1 basis point in additional growth. ${ }^{18}$ Eliminating all consumption uncertainty is valued at $1 \%$ in additional growth or more; or equivalently, the cost of all consumption uncertainty represents about half the value of long-term historical growth.

Define $\lambda(\alpha)$ as the solution to

$$
U\left(\left\{[1+\lambda(\alpha)]^{t} C_{t}\right\}\right)=U((1-\alpha)\{C\}+\alpha\{\mathfrak{C}\}),
$$

where $\left\{[1+\lambda(\alpha)]^{t} C_{t}\right\}$ is the process which has value $[1+\lambda(\alpha)]^{t} C_{t}\left(z^{t}\right)$ at time $t$, for event $z^{t}$. Notice that by definition, $\lambda(0)=0$. Assuming that $U(\cdot)$ is differentiable, differentiating both sides of (6.1), and evaluating them at $\alpha=0$, we obtain

$$
\lambda^{\prime}(0) \sum_{t \geq 1} \sum_{z^{t} \in Z^{t}} U_{z^{t}}(\{C\}) C_{t}\left(z^{t}\right) t=\sum_{t \geq 1} \sum_{z^{t} \in Z^{t}} U_{z^{t}}(\{C\})\left[\mathfrak{C}_{t}\left(z^{t}\right)-C_{t}\left(z^{t}\right)\right] .
$$

Rearranging terms, we can write this expression as

$$
\lambda^{\prime}(0)=\frac{\omega^{m}}{\sum_{t=1}^{\infty} t w_{0}\left[C_{t}\right]},
$$

where $w_{0}$ are the strip weights as in equation (4.1). We call $\sum_{t=1}^{\infty} t w_{0}\left[C_{t}\right]$ the duration of the consumption-equity, by analogy with the definition of duration in

\footnotetext{
${ }^{16}$ Note that log-normality is not required for this derivation.

${ }^{17}$ We select this high value for the riskfree rate $r_{f}$ corresponding to the long-term yield because in the random walk case, the term structure is flat.

${ }^{18}$ We thank John Cochrane for suggesting this extension.
} 
the context of coupon bonds. Thus, the trade-off between growth and fluctuations is given by the ratio of the marginal cost of fluctuations to the duration of the consumption-equity. Before presenting estimates of $\lambda^{\prime}(0)$, we notice that $\lambda$ is concave for small $\alpha$. In this case, $\lambda^{\prime}(0) \geq \lambda(\alpha) / \alpha$ for small $\alpha$; that is, the marginal cost is larger than the average cost.

Proposition 6.1. Let $U$ be increasing, concave, and twice differentiable. Then $\lambda^{\prime \prime}(0) \leq 0$.

Using our estimated pricing kernels in Tables $2 \mathrm{~A}-2 \mathrm{D}$, we can see that the duration of consumption-equity is about 50 years. With cointegration, these numbers are usually smaller; without cointegration, they are often larger. These durations can be combined with the estimates of the marginal cost of fluctuations to arrive at the trade-off between growth and cyclical variations. Based on this, eliminating business cycle fluctuations is worth less than 1 basis point in additional growth. Eliminating all consumption uncertainty is valued at $1 \%$ additional growth or more; or equivalently, the cost of all consumption uncertainty represents about half the value of long-term historical growth.

Under the random walk assumption for consumption and the pricing kernel, the duration of consumption-equity also has a particularly simple form:

$$
\sum_{t=1}^{\infty} t w_{0}\left[C_{t}\right]=\frac{1+r_{c e}}{r_{c e}-g_{c}}
$$

To get some quantitative feel for this expression, we plug in the same estimates as in Section 6.1. We consider $r_{c e}-r_{f}$ at $1.5 \%$, the riskfree rate $r_{f}=2.5 \%$, and the growth rate of consumption $g_{c}=2 \%$. In this case, the duration of consumptionequity, $\sum_{t=1}^{\infty} t w_{0}\left[C_{t}\right]$, is about 50 ; that is, $\frac{1+r_{c e}}{r_{c e}-g_{c}}=1.04 /(0.04-0.02)=52$. Thus, with the marginal cost of business cycle fluctuations for the 8-year filter usually less than 50 basis points in terms of the uniform compensation $\Omega^{\prime}(0)$, eliminating business cycles is worth less than 1 basis point in terms of additional long-term growth $\lambda^{\prime}(0)$.

\section{Summary and Conclusions}

In this paper, we have measured the cost of business cycle fluctuations using asset prices. We have used a new approach that focuses on the marginal cost of consumption fluctuations, because asset prices correspond to marginal valuations 
of market participants. We have shown that the marginal cost of consumption fluctuations corresponds to the ratio of two asset prices. We have established that the marginal cost of consumption fluctuations provides an upper bound for the benefits of reducing fluctuations completely. Our analysis shows that the equity premium and the cost of consumption uncertainty are related, but clearly distinct, both conceptually and quantitatively. The steepness of the term structure and the persistence of the shocks are two of the features that make the equity premium different from the marginal cost of consumption uncertainty.

We have estimated a pricing kernel as a way to interpolate from existing asset prices the prices of the assets that measure the marginal cost of consumption fluctuations. Our quantitative analysis highlights the importance of addressing more precisely the issue of what business cycle fluctuations correspond to. Specifically, we have estimated the marginal cost of all consumption uncertainty to be very large. Nevertheless, when we define business cycles as fluctuations that last up to 8 years, we have estimated the marginal cost of business cycles to be about one-half of a percent of lifetime consumption or less. Alternatively, the benefits of eliminating this type of fluctuation are smaller than the benefits of a permanent increase in the growth rate of consumption of 1 basis point per year. 


\section{Appendix A: Data}

The data used in this paper are annual, cover the period 1889-1997 and come mainly from two sources: Shiller (1998) and Ibbotson Associates (1998). The breakdown by time series is given below.

Consumption is real per capita consumption for nondurables and services from Shiller. We updated this series for the period after 1985 with national income and product account data from the U.S. Bureau of Economic Analysis and population data from the U.S. Bureau of the Census.

Stock prices and corresponding dividends are the Standard and Poor composite stock price indexes from Shiller.

Short-term rates are one-year returns based on 6-month commercial paper returns from Shiller, adjusted for a default premium for the period before 1926; after 1926, they are based on monthly holding periods for T-Bills from Ibbotson Associates.

The long-short yield spreads for government bonds are from Campbell (1996) for the period before 1926; after 1926, they are from Ibbotson Associates.

All series that have not been originally deflated were deflated by the producer price index from Shiller for the period before 1926; after 1926, they were deflated by the consumer price index reported in Ibbotson Associates. 


\section{Appendix B: Proofs and Details of Approximation}

Proposition 3.1. Regarding $C=\{C\}$ as a vector on $R^{\infty}$ let $\frac{\partial U(C)}{\partial C}$ and $\frac{\partial^{2} U(C)}{\partial C \partial C^{\prime}}$ be the first and second derivatives evaluated at $C$. Define $C_{\alpha}=(1-\alpha) \mathfrak{C}+\alpha C$. Then twice differentiating with respect to $\alpha$ the equation defining $\Omega$,

$$
\begin{aligned}
& \frac{\partial^{2} \Omega(\alpha)}{\partial \alpha \partial \alpha} \frac{\partial U([1+\Omega(\alpha)] C)}{\partial C} C+\left(\frac{\partial \Omega(\alpha)}{\partial \alpha} C\right)^{\prime} \frac{\partial^{2} U([1+\Omega(\alpha)] C)}{\partial C \partial C^{\prime}}\left(\frac{\partial \Omega(\alpha)}{\partial \alpha} C\right) \\
= & (\mathfrak{C}-C)^{\prime} \frac{\partial^{2} U\left(C_{\alpha}\right)}{\partial C \partial C^{\prime}}(\mathfrak{C}-C) .
\end{aligned}
$$

Taking $\alpha \downarrow 0$, since $C_{0}=[1+\Omega(0)] C=C$,

$\frac{\partial^{2} \Omega(0)}{\partial \alpha \partial \alpha} \frac{\partial U(C)}{\partial C} C=\left(\mathfrak{C}-\left[1+\frac{\partial \Omega(0)}{\partial \alpha}\right] C\right)^{\prime} \frac{\partial^{2} U(C)}{\partial C \partial C^{\prime}}\left(\mathfrak{C}-\left[1+\frac{\partial \Omega(0)}{\partial \alpha}\right] C\right) \leq 0$

where the inequality follows from the concavity of $U$. Since $U$ is increasing, $\frac{\partial^{2} \Omega(0)}{\partial \alpha \partial \alpha} \leq 0$. By concavity, $\Omega(\alpha) \leq \Omega(0)+\Omega^{\prime}(0) \alpha$ for small $\alpha$.

Proposition 3.2. If $U$ is increasing and concave in $\{C\}$, there must exist a utility function $v$ that is homogeneous of degree one, positive, and quasiconcave, and satisfies

$$
U(\{C\})=\frac{[v(\{C\})]^{1-\gamma}}{1-\gamma} .
$$

To start, we show that $\Omega(\alpha)$ is concave in $\alpha$. By homogeneity of $U$,

$$
(1+\Omega(\alpha))^{1-\gamma} \frac{[v(\{C\})]^{1-\gamma}}{1-\gamma}=\frac{[v((1-\alpha)\{C\}+\alpha\{\mathfrak{C}\})]^{1-\gamma}}{1-\gamma} .
$$

Thus, after multiplying by $(1-\gamma)$, taking the $1 /(1-\gamma)$ power, and dividing by $v(\{C\})$ on both sides, we obtain that

$$
1+\Omega(\alpha)=\frac{v((1-\alpha)\{C\}+\alpha\{\mathfrak{C}\})}{v(\{C\})}
$$

Since $v(\cdot)$ is positive, quasiconcave and homogeneous of degree one, it is concave. With $(1-\alpha)\{C\}+\alpha\{\mathfrak{C}\}$ linear in $\alpha, v(\cdot)$ is also concave in $\alpha$; thus, $\Omega(\alpha)$ is concave. Now we use the concavity to obtain the desired relationships,

$$
\Omega(1)=\Omega(0)+\int_{0}^{1} \Omega^{\prime}(\alpha) d \alpha \leq \Omega^{\prime}(0)
$$


where the inequality uses $\Omega(0)=0$, the concavity of $\Omega$, and that $\alpha \leq 1$.

Proposition 3.3. From the concavity of $u$ and the definition of $\mathfrak{C}$,

$$
\begin{aligned}
U((1-\alpha)\{C\}+\alpha\{\mathfrak{C}\}) & =E\left[u\left((1-\alpha) C_{1}+\alpha \mathfrak{C}_{1},(1-\alpha) C_{2}+\alpha \mathfrak{C}_{2}, \ldots,(1-\alpha) C_{t}+\alpha \mathfrak{C}_{t}, \ldots\right)\right] \\
& \leq u\left(\mathfrak{C}_{1}, \mathfrak{C}_{2}, \ldots, \mathfrak{C}_{1}, \ldots\right)=U(\{\mathfrak{C}\})
\end{aligned}
$$

for any $\alpha$. By the definition of $\Omega$ and the monotonicity of $U, \Omega$ has to reach a maximum on $\alpha=1$. Thus $\Omega^{\prime}(1)=0$, and $\Omega(\alpha)$ is concave for $\alpha$ close to 1 .

Proposition 3.4. To simplify the notation, denote $\Omega^{\prime}(0, \sigma)$ by $\Omega^{\prime}(\sigma)$ and $\Omega(0, \sigma)$ by $\Omega(\sigma)$. Consider the total cost $\Omega(\sigma)$. We use Taylor expansions for the right and left hand side of the definition of the total cost to find an expression for $\Omega(\sigma)$. The utility $u(\bar{C})$ can be approximated around $\bar{C}(1+\Omega(\sigma))$ as

$$
u(\bar{C})=u(\bar{C}(1+\Omega(\sigma)))-u^{\prime}\left(\bar{C}_{\sigma}\right) \bar{C} \Omega(\sigma),
$$

where $\bar{C}_{\sigma} \in[\bar{C}, \bar{C}(1+\Omega(\sigma))]$ and $\lim _{\sigma \rightarrow 0} \bar{C}_{\sigma}=\bar{C}$. The utility $u(\bar{C}(1+\sigma \varepsilon)(1+\Omega(\sigma)))$ can be approximated around $\bar{C}(1+\sigma \varepsilon)$ for each $\varepsilon$ as

$$
\begin{aligned}
& u(\bar{C}(1+\sigma \varepsilon)(1+\Omega(\sigma))) \\
= & u(\bar{C}(1+\Omega(\sigma)))+u^{\prime}(\bar{C}(1+\Omega(\sigma)))(1+\Omega(\sigma)) \bar{C} \sigma \varepsilon \\
& +\frac{1}{2} u^{\prime \prime}(\bar{C}(1+\Omega(\sigma)))((1+\Omega(\sigma)) \bar{C} \sigma \varepsilon)^{2} \\
& +\frac{1}{6} u^{\prime \prime \prime}(\bar{C}(1+\Omega(\sigma)))((1+\Omega(\sigma)) \bar{C} \sigma \varepsilon)^{3}+\frac{1}{24} u^{\prime \prime \prime \prime}\left(\bar{C}_{\sigma}(\varepsilon)\right)(\bar{C} \sigma \varepsilon)^{4},
\end{aligned}
$$

where $\bar{C}_{\sigma}(\varepsilon) \in[\bar{C}(1+\sigma \varepsilon), \bar{C}(1+\sigma \varepsilon)(1+\Omega(\sigma))]$ for positive $\varepsilon$ and $[\bar{C}(1+\sigma \varepsilon)(1+\Omega(\sigma)), \bar{C}(1+\sigma \varepsilon)]$ for negative $\varepsilon$, and $\lim _{\sigma \rightarrow 0} \bar{C}_{\sigma}(\varepsilon)=\bar{C}$. Then taking expected values,

$$
\begin{aligned}
& E[u(\bar{C}(1+\sigma \varepsilon)(1+\Omega(\sigma)))] \\
= & u(\bar{C}(1+\Omega(\sigma)))+\frac{1}{2} u^{\prime \prime}(\bar{C}(1+\Omega(\sigma))) E[(1+\Omega(\sigma)) \bar{C} \sigma \varepsilon]^{2} \\
& +\frac{1}{6} u^{\prime \prime \prime}(\bar{C}(1+\Omega(\sigma))) E[(1+\Omega(\sigma)) \bar{C} \sigma \varepsilon]^{3}+o\left(\sigma^{3}\right) .
\end{aligned}
$$

Using the definition of $\Omega(\sigma)$, we now have

$$
\begin{aligned}
& -u^{\prime}\left(\bar{C}_{\sigma}\right) \bar{C} \Omega(\sigma) \\
= & \frac{1}{2} u^{\prime \prime}(\bar{C}(1+\Omega(\sigma))) E[(1+\Omega(\sigma)) \bar{C} \sigma \varepsilon]^{2} \\
& +\frac{1}{6} u^{\prime \prime \prime}(\bar{C}(1+\Omega(\sigma))) E[(1+\Omega(\sigma)) \bar{C} \sigma \varepsilon]^{3}+o\left(\sigma^{3}\right) .
\end{aligned}
$$


As an intermediate step, we first show that

$$
\Omega(\sigma)=\frac{\sigma^{2}}{2} \frac{\bar{C} u^{\prime \prime}(\bar{C})}{u^{\prime}(\bar{C})} E \varepsilon^{2}+o\left(\sigma^{2}\right) \equiv k \sigma^{2}+o\left(\sigma^{2}\right)
$$

which follows since $\Omega(\sigma) \rightarrow 0$, and $\bar{C}_{\sigma} \rightarrow \bar{C}$ as $\sigma \rightarrow 0$. Now we show that

$$
u^{\prime}\left(\bar{C}_{\sigma}\right) \bar{C} \Omega(\sigma)=u^{\prime}(\bar{C}) \bar{C} \Omega(\sigma)+o\left(\sigma^{3}\right)
$$

which follows from

$$
u^{\prime}\left(\bar{C}_{\sigma}\right) \bar{C} \Omega(\sigma)=u^{\prime}(\bar{C}) \bar{C} \Omega(\sigma)+u^{\prime \prime}(C(\sigma)) \bar{C} \Omega(\sigma)\left(\bar{C}_{\sigma}-\bar{C}\right)
$$

for some $C(\sigma) \in\left[\bar{C}, \bar{C}_{\sigma}\right]$. Recall that by definition of $\bar{C}_{\sigma}$

$$
0 \leq\left(\bar{C}_{\sigma}-\bar{C}\right) \leq \bar{C} \Omega(\sigma)
$$

so

$$
\bar{C} \Omega(\sigma)\left(\bar{C}_{\sigma}-\bar{C}\right) \leq(\bar{C} \Omega(\sigma))^{2}=\sigma^{4} k+o\left(\sigma^{3}\right),
$$

for some constant $k$. Also, we show that

$$
\begin{aligned}
& u^{\prime \prime}(\bar{C}(1+\Omega(\sigma)))(1+\Omega(\sigma))^{2} \\
= & u^{\prime \prime}(\bar{C})+o\left(\sigma^{1}\right),
\end{aligned}
$$

which follows from

$$
\begin{aligned}
& u^{\prime \prime}(\bar{C}(1+\Omega(\sigma)))(1+\Omega(\sigma))^{2} \\
= & u^{\prime \prime}(\bar{C}(1+\Omega(\sigma)))\left(1+2 k \sigma^{2}+o\left(\sigma^{2}\right)+o\left(\sigma^{4}\right)+k^{2} \sigma^{4}\right)
\end{aligned}
$$

and from

$$
\begin{aligned}
u^{\prime \prime}(\bar{C}(1+\Omega(\sigma))) & =u^{\prime \prime}(\bar{C})+u^{\prime \prime \prime}(\bar{C}(\sigma)) \bar{C} \Omega(\sigma) \\
& =u^{\prime \prime}(\bar{C})+u^{\prime \prime \prime}(\bar{C}(\sigma))\left(k \sigma^{2}+o\left(\sigma^{2}\right)\right) .
\end{aligned}
$$

Using these last two results we can write (7.1) as

$$
\begin{aligned}
& -u^{\prime}(\bar{C}) \bar{C} \Omega(\sigma)+o\left(\sigma^{3}\right) \\
= & \frac{1}{2} u^{\prime \prime}(\bar{C}) E[\bar{C} \sigma \varepsilon]^{2}+\frac{\sigma^{2} o(\sigma)}{2} E[\bar{C} \varepsilon]^{2}+\frac{1}{6} u^{\prime \prime \prime}(\bar{C}(1+\Omega(\sigma))) E[\bar{C} \sigma \varepsilon]^{3}+o\left(\sigma^{3}\right) .
\end{aligned}
$$


Dividing by $u^{\prime}(\bar{C}) \bar{C}$ on both sides, we obtain that

$$
\Omega(\sigma)=-\frac{\sigma^{2}}{2} \frac{\bar{C} u^{\prime \prime}(\bar{C})}{u^{\prime}(\bar{C})} E \varepsilon^{2}-\frac{\sigma^{3}}{6} \frac{\bar{C}^{2} u^{\prime \prime \prime}(\bar{C})}{u^{\prime}\left(\bar{C}_{\sigma}\right)} E \varepsilon^{3}+o\left(\sigma^{3}\right) .
$$

We apply similar arguments to the expression for the marginal cost $\Omega^{\prime}(\sigma)$.

Proposition 3.6. The core of this proof is similar to the one-period case, so we only provide a sketch here. Consider the total cost $\Omega(\sigma)$ defined as

$$
\sum_{t=1}^{T} \beta^{t} E_{0}\left[u\left(\bar{C}_{t}\left(1+\sigma \varepsilon_{t}\right)(1+\Omega(\sigma))\right)\right]=\sum_{t=1}^{T} \beta^{t} u\left(\bar{C}_{t}\right)
$$

Using exactly the same arguments as for the one-period case, we can write $u\left(\bar{C}_{t}\right)$ for each period $t$ as

$$
u\left(\bar{C}_{t}\right)=u\left(\bar{C}_{t}(1+\Omega(\sigma))\right)-u^{\prime}\left(\bar{C}_{t, \sigma}\right) \bar{C} \Omega(\sigma),
$$

where $\lim _{\sigma \rightarrow 0} \bar{C}_{t, \sigma}=\bar{C}_{t}$ for all $t$. Also for each $t$ we can write $E_{0}\left[u\left(C_{t}(1+\Omega(\sigma))\right)\right]$ as

$$
\begin{aligned}
E_{0}\left[u\left(\bar{C}_{t}\left(1+\sigma \varepsilon_{t}\right)(1+\Omega(\sigma))\right)\right]= & u\left(\bar{C}_{t}(1+\Omega(\sigma))\right)+\frac{1}{2} u^{\prime \prime}\left(\bar{C}_{t}(1+\Omega(\sigma))\right) E_{0}\left[\bar{C}_{t} \sigma \varepsilon_{t}\right]^{2} \\
& +\frac{1}{6} u^{\prime \prime \prime}\left(\bar{C}_{t}(1+\Omega(\sigma))\right) E_{0}\left[\bar{C}_{t} \sigma \varepsilon_{t}\right]^{3}+o_{t}\left(\sigma^{3}\right) .
\end{aligned}
$$

Replacing the expansions in the definition of $\Omega(\sigma)$, yields that

$$
\begin{aligned}
& \sum_{t=1}^{T} \beta^{t}\left\{u\left(\bar{C}_{t}(1+\Omega(\sigma))\right)-\Omega(\sigma) u^{\prime}\left(\bar{C}_{t, \sigma}\right) \bar{C}_{t}\right\} \\
= & \sum_{t=1}^{T} \beta^{t}\left\{\begin{array}{c}
u\left(\bar{C}_{t}(1+\Omega(\sigma))\right)+\frac{1}{2} u^{\prime \prime}\left(\bar{C}_{t}(1+\Omega(\sigma))\right) E_{0}\left[\bar{C}_{t} \sigma \varepsilon_{t}\right]^{2} \\
+\frac{1}{6} u^{\prime \prime \prime}\left(\bar{C}_{t}(1+\Omega(\sigma))\right) E_{0}\left[\bar{C}_{t} \sigma \varepsilon_{t}\right]^{3}+o_{t}\left(\sigma^{3}\right)
\end{array}\right\},
\end{aligned}
$$

and simplifying yields that

$$
\begin{aligned}
& \Omega(\sigma) \sum_{t=1}^{T} \beta^{t} u^{\prime}\left(\bar{C}_{t, \sigma}\right) \bar{C}_{t} \\
= & \sum_{t=1}^{T} \beta^{t}\left\{-\frac{1}{2} u^{\prime \prime}\left(\bar{C}_{t}(1+\Omega(\sigma))\right) E_{0}\left[\bar{C}_{t} \sigma \varepsilon_{t}\right]^{2}-\frac{1}{6} u^{\prime \prime \prime}\left(\bar{C}_{t}(1+\Omega(\sigma))\right) E_{0}\left[\bar{C}_{t} \sigma \varepsilon_{t}\right]^{3}+o_{t}\left(\sigma^{3}\right)\right\} .
\end{aligned}
$$


By applying the same procedure as for the one-period case, we get that

$$
\begin{aligned}
& \Omega(\sigma) \sum_{t=1}^{T} \beta^{t} u^{\prime}\left(\bar{C}_{t}\right) \bar{C}_{t} \\
= & \sum_{t=1}^{T} \beta^{t}\left\{-\frac{1}{2} u^{\prime \prime}\left(\bar{C}_{t}\right) E_{0}\left[\bar{C}_{t} \sigma \varepsilon_{t}\right]^{2}-\frac{1}{6} u^{\prime \prime \prime}\left(\bar{C}_{t}\right) E_{0}\left[\bar{C}_{t} \sigma \varepsilon_{t}\right]^{3}+o_{t}\left(\sigma^{3}\right)\right\} .
\end{aligned}
$$

Rearranging, we get that

$$
\Omega(\sigma)=\sum_{t=1}^{T} \bar{w}_{t}\left\{-\frac{1}{2} \frac{\bar{C}_{t} u^{\prime \prime}\left(\bar{C}_{t}\right)}{u^{\prime}\left(\bar{C}_{t}\right)} \sigma^{2} E_{0}\left[\varepsilon_{t}\right]^{2}-\frac{1}{6} \frac{\bar{C}_{t}^{2} u^{\prime \prime \prime}\left(\bar{C}_{t}\right)}{u^{\prime}\left(\bar{C}_{t}\right)} \sigma^{3} E_{0}\left[\varepsilon_{t}\right]^{3}\right\}+o\left(\sigma^{3}\right),
$$

where

$$
\bar{w}_{t}=\frac{\beta^{t} u^{\prime}\left(\bar{C}_{t}\right) \bar{C}_{t}}{\sum_{j=1}^{T} \beta^{t} u^{\prime}\left(\bar{C}_{j}\right) \bar{C}_{j}}
$$

Analogous steps are applied for the marginal cost.

Proposition 3.9. Notice that the left hand side of (3.3) and (3.4) are the same. Consider any arbitrary $\alpha$. By definition of envy, making $\{\hat{C}\}=(1-\alpha)\{C\}+$ $\alpha\{\mathfrak{C}\}$, we obtain the desired result.

Proposition 4.1. Using the definition of the weights $w_{0}\left[C_{t}\right]$ in equation (4.1), multiplying and dividing by $E_{0}\left(C_{t}\right)$, and using the definitions of $R_{0, t}\left[C_{t}\right]$ and $R_{0, t}\left[1_{t}\right]$, we have that

$$
\begin{aligned}
1+\omega_{0}^{m} & =\frac{\sum_{t=1}^{\infty} V_{0}\left[E_{0}\left(C_{t}\right)\right]}{\sum_{t=1}^{\infty} V_{0}\left[C_{t}\right]} \\
& =\sum_{t=1}^{\infty} w_{0}\left[C_{t}\right]\left(\frac{V_{0}\left[E_{0}\left(C_{t}\right)\right]}{V_{0}\left[C_{t}\right]}\right) \\
& =\sum_{t=1}^{\infty} w_{0}\left[C_{t}\right]\left(\frac{E_{0}\left(C_{t}\right)}{V_{0}\left[C_{t}\right]} / \frac{E_{0}\left(C_{t}\right)}{V_{0}\left[E_{0}\left(C_{t}\right)\right]}\right) \\
& =\sum_{t=1}^{\infty} w_{0}\left[C_{t}\right]\left(R_{0, t}\left[C_{t}\right] / R_{0, t}\left[1_{t}\right]\right) .
\end{aligned}
$$


Using the definition of the equity premium, the decomposition of the price of equity as the sum of the strips, the definitions of $w_{0}\left[C_{t}\right]$, and the definition of excess one-period holding returns, we have that

$$
\begin{aligned}
1+\nu_{0} & =\frac{E_{0}\left(V_{1}\left[\left\{C_{t}\right\}\right]\right)}{V_{0}\left[\left\{C_{t}\right\}\right]} / \frac{1}{V_{0}\left(1_{1}\right)} \\
& =\sum_{t=1}^{\infty} w_{0}\left[C_{t}\right]\left(\frac{E_{0}\left(R_{0,1}\left[C_{t}\right]\right)}{R_{0,1}\left[1_{1}\right]}\right) .
\end{aligned}
$$

Proposition 5.1. Notice that the random walk assumption implies that interest rates are constant, since

$$
E_{t}\left(\frac{M_{t+1}}{M_{t}}\right)=E\left(\frac{M_{t+1}}{M_{t}}\right)
$$

Hence, the term premium is zero. Then

$$
\begin{aligned}
\frac{E_{t}\left(R_{t+1}\left[D_{t+s}\right]\right)}{E_{t} R_{t+1}\left(1_{t+1}\right)} & =\frac{E_{t}\left(R_{t+1}\left[D_{t+s} / D_{t}\right]\right)}{E_{t} R_{t+1}\left(1_{t+1}\right)}=E_{t}\left(\beta \frac{M_{t+1}}{M_{t}}\right) \frac{E_{t}\left(\frac{D_{t+1}}{D_{t}} E_{t+1}\left[\beta^{s-1} \frac{M_{t+s}}{M_{t+1}} \frac{D_{t+s}}{D_{t+1}}\right]\right)}{E_{t}\left[\beta^{s} \frac{M_{t+s}}{M_{t}} \frac{D_{t+1}}{D_{t}} \frac{D_{t+s}}{D_{t+1}}\right]} \\
& =\exp \left(-\operatorname{cov}_{t}\left(\log \frac{M_{t+1}}{M_{t}}, E_{t}\left(\log \frac{D_{t+s}}{D_{t}}\right)\right)\right) \\
& =\exp \left(-\operatorname{cov}_{t}\left(m_{t+1}, d_{t+1}\right)\right) \text { for all } s \geq 1,
\end{aligned}
$$

where the first inequality follows since $D_{t}$ is known at $t$, the second from the definition of returns and the pricing kernel, the third from the log-normality of $D$ and $M$ as in (4.5) since the term premium is zero, and the fourth follows by the random walk assumptions. From this we obtain that the excess expected return of a stock paying $\left\{D_{t+s}\right\}$ is

$$
\frac{E_{t}\left(R_{t+1}\left[\left\{D_{t+s}\right\}_{s=1}^{\infty}\right]\right)}{E_{t} R_{t+1}\left(1_{t+1}\right)}=\sum_{s=1}^{\infty} w\left(\left[D_{t+s}\right]\right) \frac{E_{t}\left(R_{t+1}\left[D_{t+s}\right]\right)}{E_{t} R_{t+1}\left(1_{t+1}\right)}=\frac{E_{t}\left(R_{t+1}\left[D_{t+s}\right]\right)}{E_{t} R_{t+1}\left(1_{t+1}\right)}
$$

where the weights $w\left[D_{t+s}\right]=V_{t}\left[\left\{D_{t+s}\right\}\right] / V\left[\left\{D_{t+j}\right\}_{j=1}^{\infty}\right]$ sum to one. For this stock, the price/dividend ratio is given by

$$
V_{t}\left[\left\{D_{t+s}\right\}_{s=1}^{\infty}\right] / D_{t}=\sum_{s=1}^{\infty} \beta^{s} E_{t}\left[\frac{M_{t+s}}{M_{t}} \frac{D_{t+s}}{D_{t}}\right]=\sum_{s=1}^{\infty} \prod_{i=1}^{s} E_{t}\left[\beta \frac{M_{t+i}}{M_{t+i-1}} \frac{D_{t+i}}{D_{t+i-1}}\right]
$$




$$
=\sum_{s=1}^{\infty}\left(E\left[\beta \frac{M_{t+1}}{M_{t}} \frac{D_{t+1}}{D_{t}}\right]\right)^{s}=\frac{E\left[\beta \frac{M_{t+1}}{M_{t}} \frac{D_{t+1}}{D_{t}}\right]}{1-E\left[\beta \frac{M_{t+1}}{M_{t}} \frac{D_{t+1}}{D_{t}}\right]}
$$

where the third inequality uses the random walk assumption. Finally, notice that for each asset $i$ we have that

$$
\log E_{t}\left(R_{t+1, i}\right)=-\operatorname{cov}_{t}\left(\Delta m_{t+1}, \Delta d_{t+1}^{i}\right)=-\operatorname{cov}\left(l \cdot \varepsilon_{t+1}, \Delta d_{t+1}^{i}\right)
$$

Proposition 5.2. We start by finding an expression for $\tau(t)$. Notice that using log-normality, we get

$E_{0}\left(\mathfrak{C}_{t}\right)=\exp (\tau) E_{0} \exp \left(\sum_{i=1}^{K} a_{i} c_{t-j}\right)=\exp (\tau(t)) \exp \left(\sum_{i=1}^{K} a_{i} E_{0}\left(c_{t-j}\right)+\frac{1}{2} \operatorname{var}_{0}\left(\sum_{i=1}^{K} a_{i} c_{t-j}\right)\right)$

and

$$
E_{0}\left(C_{t}\right)=E_{0} \exp \left(c_{t}\right)=\exp \left(E_{0} c_{t}+\frac{1}{2} \operatorname{var}_{0}\left(c_{t}\right)\right)
$$

Thus, $\tau(t)$ solves $E_{0}\left(\mathfrak{C}_{t}\right)=E_{0}\left(C_{t}\right)$. Therefore,

$$
\tau(t)=\exp \left(E_{0} c_{t}-\sum_{i=1}^{K} a_{i} E_{0}\left(c_{t-j}\right)+\frac{1}{2} \operatorname{var}_{0}\left(c_{t}\right)-\frac{1}{2} \operatorname{var}_{0}\left(\sum_{i=1}^{K} a_{i} c_{t-j}\right)\right) .
$$

Using the definition of the pricing kernel and lognormality

$$
\begin{aligned}
\frac{V_{0}\left[\mathfrak{C}_{t}\right]}{V_{0}\left[C_{t}\right]}= & \frac{E_{0} \exp \left[\tau(t)+E_{0} m_{t}-m_{0}+\sum_{j=0}^{K} a_{j} c_{t-j}\right]}{E_{0} \exp \left[m_{t}-m_{0}+c_{t}\right]} \\
= & \frac{\exp \left[\tau(t)+E_{0}\left(m_{t}-m_{0}+\sum_{j=0}^{K} a_{j} c_{t-j}\right)+\frac{1}{2} \operatorname{var}_{0}\left(m_{t}\right)\right]}{\exp \left[E_{0}\left(m_{t}-m_{0}+c_{t}\right)+\frac{1}{2} \operatorname{var}_{0}\left(m_{t}\right)+\frac{1}{2} \operatorname{var}_{0}\left(c_{t}\right)+\operatorname{cov}_{0}\left(m_{t}, c_{t}\right)\right]} \\
& \times \exp \left[\frac{1}{2} \operatorname{var}_{0}\left(\sum_{j=0}^{K} a_{j} c_{t-j}\right)+\operatorname{cov}_{0}\left(m_{t}, \sum_{j=0}^{K} a_{j} c_{t-j}\right)\right] \\
= & \exp \left[\begin{array}{c}
\tau(t)+\frac{1}{2} \operatorname{var}_{0}\left(\sum_{j=0}^{K} a_{j} c_{t-j}\right)-\frac{1}{2} \operatorname{var}_{0}\left(c_{t}\right) \\
+\operatorname{cov}_{0}\left(m_{t}, \sum_{j=0}^{K} a_{j} c_{t-j}\right)-\operatorname{cov}_{0}\left(m_{t}, c_{t}\right)
\end{array}\right] .
\end{aligned}
$$


By definition of $\tau(t)$, we get that

$$
\frac{V_{0}\left[\mathfrak{C}_{t}\right]}{V_{0}\left[C_{t}\right]}=\exp \left[\operatorname{cov}_{0}\left(m_{t}, \sum_{j=0}^{K} a_{j} c_{t-j}\right)-\operatorname{cov}_{0}\left(m_{t}, c_{t}\right)\right]
$$

By direct computation in the random walk case we get that

$$
\begin{aligned}
& =\exp \left[\operatorname{cov}_{0}\left(m_{t}, \sum_{j=0}^{K} a_{j} c_{t-j}\right)-\operatorname{cov}_{0}\left(m_{t}, c_{t}\right)\right] \\
& =\exp \left[\operatorname{cov}_{0}\left(\sum_{k=(t-K)}^{t} \varepsilon_{k}^{m}, \sum_{k=(t-K)}^{t} \sum_{j=0}^{K} a_{j} \varepsilon_{k-j}^{c}\right)-\operatorname{cov}_{0}\left(\sum_{k=(t-K)}^{t} \varepsilon_{k}^{m}, \sum_{k=(t-K)}^{t} \varepsilon_{k-j}^{c}\right)\right] \\
& =\exp \left[\begin{array}{c}
\operatorname{cov}_{0}\left(\varepsilon_{t}^{m}, a_{0} \varepsilon_{t}^{c}\right)-\operatorname{cov}_{0}\left(\varepsilon_{t}^{m}, \varepsilon_{t}^{c}\right)+ \\
\operatorname{cov}_{0}\left(\varepsilon_{t-1}^{m},\left(a_{0}+a_{1}\right) \varepsilon_{t-1}^{c}\right)-\operatorname{cov}_{0}\left(\varepsilon_{t-K-1}^{m}, \varepsilon_{t-1}^{c}\right)+\cdots+ \\
\operatorname{cov}\left(\varepsilon_{t-K}^{m},\left(a_{0}+a_{1}+\cdots+a_{K}\right) \varepsilon_{t-K-1}^{c}\right)-\operatorname{cov}_{0}\left(\varepsilon_{t-K}^{m}, \varepsilon_{t-K}^{c}\right)
\end{array}\right] \\
& =\exp \left[\sigma_{\Delta c \Delta m}\left[a_{0}-1+\left(a_{0}+a_{1}\right)-1+\cdots+\left(a_{0}+a_{1}+\cdots+a_{K}\right)-1\right]\right] \text {, }
\end{aligned}
$$

where $\varepsilon^{m}$ and $\varepsilon^{c}$ are the innovations of the log of the pricing kernel and the log of consumption. While the first step uses the fact that the first $t-K$ shocks are common to both covariances, the second separates the covariances, and the third reorders and uses the result that for the last term the sum of all the $\alpha^{\prime} s$ equals 1 . Finally, by Proposition 5.1, we have that

$$
\frac{E\left(R_{1}\left[C_{t}\right]\right)}{R_{f}}=\exp \left(-\operatorname{cov}\left(\Delta m_{t}, \Delta c_{t}\right)\right) \equiv \exp \left(-\sigma_{\Delta c, \Delta m}\right)
$$

which, combined with the previous equality, finishes the proof.

Proposition 5.3. This proof is a straightforward variation of the proof of Proposition 5.2.

Proposition 5.4. Direct computation shows that

$$
\begin{aligned}
m\left(a^{k+1}\right)= & \left(a_{0}^{K+1}-1\right)+\left(a_{0}^{K+1}+a_{1}^{K+1}-1\right)+\left(a_{0}^{K+1}+a_{1}^{K+1}+a_{2}^{K}-1\right) \\
& +\cdots+\left(a_{0}^{K+1}+a_{1}^{K+1}+\cdots+a_{K+1}^{K}-1\right) \\
= & -1+\left(a_{0}^{K}-1\right)+\left(a_{0}^{K}+a_{1}^{K}-1\right)+\cdots+\left(a_{0}^{K+1}+a_{1}^{K+1}+\cdots+a_{K}^{K}-1\right) \\
= & m\left(a^{K}\right)+1 .
\end{aligned}
$$


Proposition 6.1. Regarding $C=\{C\}$ as a vector on $R^{\infty}$, let $\frac{\partial U(C)}{\partial C}$ and $\frac{\partial^{2} U(C)}{\partial C \partial C^{\prime}}$ be its first and second derivatives evaluated at $C$. Define $C_{\alpha}=(1-\alpha) \mathfrak{C}+$ $\alpha C$, and let $\left(C_{t}[1+\lambda(\alpha)]^{t}\right)$ be the vector where each component consists of $C_{t}\left(z^{t}\right)[1+\lambda(\alpha)]^{t}$. Then twice differentiating with respect to $\alpha$ the equation defining $\lambda$, we get that

$$
\begin{aligned}
& \quad \frac{\partial \lambda(\alpha)}{\partial \alpha} \frac{\partial U\left(\left([1+\lambda(\alpha)]^{t} C_{t}\right)\right)}{\partial C}\left(C_{t}[1+\lambda(\alpha)]^{t-1} t\right)=\frac{\partial U\left(C_{\alpha}\right)}{\partial C}(\mathfrak{C}-C), \\
& \quad \frac{\partial^{2} \lambda(\alpha)}{\partial \alpha \partial \alpha} \frac{\partial U\left(\left([1+\lambda(\alpha)]^{t} C_{t}\right)\right)}{\partial C}\left(C_{t}[1+\lambda(\alpha)]^{t-1} t\right)+ \\
& \quad\left[\frac{\partial \lambda(\alpha)}{\partial \alpha}\right]^{2} \frac{\partial U\left(\left([1+\lambda(\alpha)]^{t} C_{t}\right)\right)}{\partial C}\left(C_{t}[1+\lambda(\alpha)]^{t-2} t(t-1)\right) \\
& \quad+\left(\frac{\partial \lambda(\alpha)}{\partial \alpha}\left(C_{t}[1+\lambda(\alpha)]^{t-1} t\right)\right)^{\prime} \frac{\partial^{2} U\left(\left([1+\lambda(\alpha)]^{t} C_{t}\right)\right)}{\partial C \partial C^{\prime}}\left(\frac{\partial \lambda(\alpha)}{\partial \alpha}\left(C_{t}[1+\lambda(\alpha)]^{t-1} t\right)\right) \\
& =(\mathfrak{C}-C)^{\prime} \frac{\partial^{2} U\left(C_{\alpha}\right)}{\partial C \partial C^{\prime}}(\mathfrak{C}-C) .
\end{aligned}
$$

Taking $\alpha \downarrow 0$ since $C_{\alpha}=C$ and $\lambda(0)=0$, we get that

$$
\begin{aligned}
& \frac{\partial^{2} \lambda(0)}{\partial \alpha \partial \alpha} \frac{\partial U(C)}{\partial C}\left(C_{t} t\right)=-\left[\frac{\partial \lambda(\alpha)}{\partial \alpha}\right]^{2} \frac{\partial U(C)}{\partial C}\left(C_{t} t(t-1)\right) \\
& +\left(\mathfrak{C}-C-\frac{\partial \lambda(\alpha)}{\partial \alpha}\left(C_{t} t\right)\right)^{\prime} \frac{\partial^{2} U(C)}{\partial C \partial C^{\prime}}\left(\mathfrak{C}-C-\frac{\partial \lambda(\alpha)}{\partial \alpha}(C t)\right) \leq 0
\end{aligned}
$$

where the inequality follows from the concavity of $U$ and the monotonicity of $U$. Since $U$ is increasing, then $\frac{\partial^{2} \lambda(0)}{\partial \alpha \partial \alpha} \leq 0$.

Details about approximations made with the log-linear system: Bias in the trend

In Section 5.3, we define the trend as a one-sided moving average of current and past consumption, $\ln \left(\mathfrak{c}_{t}\right)=\sum_{k=0}^{K} a_{k} \cdot c_{t-k}$. With this definition of the trend $\mathfrak{C}$, $E_{0}\left(C_{t}\right) \neq E_{0}\left(\mathfrak{C}_{t}\right)$, because $\mathfrak{C}$ is a nonlinear function of $C$. We show here that this bias is quantitatively negligible for our applications.

Assuming we want to impose as a constraint that the conditional means of the trend and of consumption are equal, then we must do the following. Based on our definition of the marginal cost we have

$$
1+\omega_{0}^{m}=\frac{\sum_{t=1}^{\infty} V_{0}\left[X_{t}\right]}{\sum_{t=1}^{\infty} V_{0}\left[C_{t}\right]}=\sum_{t=1}^{\infty} w_{t} \frac{V_{0}\left[X_{t}\right]}{V_{0}\left[C_{t}\right]}
$$


where $w_{t} \equiv V_{0}\left[C_{t}\right] / \sum_{t=1} V_{0}\left[C_{t}\right]$. Assume we want to adjust every strip of the trend, so that

$$
1+\omega_{0}^{m}=\sum_{t=1}^{\infty} w_{t} \frac{V_{0}\left[X_{t}\right] \phi(t)}{V_{0}\left[C_{t}\right]}
$$

If we want to make the conditional means equal, we need that

$$
\phi(t)=\frac{E_{0}\left[C_{t}\right]}{E_{0}\left[X_{t}\right]} .
$$

In this case, $\phi(t)$ is a state-dependent adjustment.

The random walk case can provide an idea of the order of magnitude of the adjustment; it is easy to compute and close to the cases analyzed in the paper. In this case, simple algebra gives that for $t>K$,

$$
\phi(t)=\frac{E_{0} C_{t}}{E_{0} X_{t}}=e^{\left[1-\left(\alpha_{0}\right)^{2}+1-\left(\alpha_{0}+\alpha_{1}\right)^{2}+1-\left(\alpha_{0}+\alpha_{1}+\alpha_{2}\right)^{2}+\ldots+1-\left(\alpha_{0}+\ldots+\alpha_{J}\right)^{2}\right] \frac{\sigma_{c}^{2}}{2}} \geq 1 .
$$

In words, expected consumption is larger because consumption is more volatile than the trend, given that the trend depends partially on earlier realizations that have a lower variance. For the first few periods where $t \leq K$, the terms are a bit different. They will coincide if we take the expectations conditional on time $-K$, so that the lags of the trend are not known; otherwise, they will depend on recent realizations of consumption.

For the coefficient values implied by the 8-year filter in the paper, based on $\sigma_{c}^{2}=0.0328^{2}$ and $\sigma_{c}^{2}=0.0115^{2}$ corresponding to the variance of the consumption growth rate for the period 1889-1997 and 1954-97 respectively, we have that

$$
\exp \left(0.5752 \frac{0.0328^{2}}{2}\right)=1.000309
$$

and

$$
\exp \left(0.5752 \frac{0.0115^{2}}{2}\right)=1.000038
$$

That is, the bias is barely 3 basis points with consumption for 1889-1997, and about one third of a basis point with consumption for 1954-97. 


\section{References}

[1] Abel, Andrew B., 1999, Risk Premia and Term Premia in General Equilibrium, Journal of Monetary Economics, 43, 3-33.

[2] Alvarez, Fernando and Jermann, Urban J., 2000, Why Pricing Kernels Should Not Be Stationary, http://finance.wharton.upenn.edu/ $\sim$ jermann/research.html.

[3] Atkeson, Andrew and Phelan, Christopher, 1994, Reconsidering the Costs of Business Cycles with Incomplete Markets, NBER Macroeconomics Annual 1994. Fischer, Stanley; Rotemberg, Julio J., eds., Cambridge: MIT Press, 187-207.

[4] Baxter, Marianne and King, Robert G., 1999, Measuring Business Cycles: Approximate Band-Pass Filters for Economic Time Series, Review of Economics and Statistics, 75-93.

[5] _______ , 1998, Band-Pass Filters for Financial and Current Analysis, University of Virginia.

[6] Burns, Arthur F. and Mitchell, Wesley C., 1946, Measuring Business Cycles, New York, N.Y.: National Bureau of Economic Research.

[7] Campbell, John Y., 1986, Bond and Stock Returns in a Simple Exchange Model, Quarterly Journal of Economics, 785-803.

[8] Campbell, John Y., 1996, Understanding Risk and Return, Journal of Political Economy, 104(2), April, 298-345.

[9] Campbell, John Y. and Cochrane John H., 1995, By Force of Habit: A Consumption-Based Explanation of Aggregate Stock Market Behavior, NBER Working Paper No. 4995, University of Chicago.

[10] Constantinides, George, M., 1990, Habit Formation: A Resolution of the Equity Premium Puzzle, Journal of Political Economy, 98, 519-543.

[11] Dolmas, Jim, 1998, Risk Preferences and the Welfare Cost of Business Cycles, Review of Economic Dynamics 1, 646-676. 
[12] Epstein, Larry G. and Zin, Stanley E., 1991, Substitution, Risk Aversion, and the Temporal Behavior of Consumption and Asset Returns: An Empirical Analysis, Journal of Political Economy 99(2), 263-286.

[13] Hansen, Lars Peter, Sargent, Thomas J. and Tallarini, Thomas D., 1999, Robust Permanent Income and Pricing, Review of Economic Studies, 873928

[14] Krusell, Per, and Smith Anthony A. Jr., 1999, On the Welfare Effects of Eliminating Business Cycles, Review of Economic Dynamics, 245-72.

[15] Jermann, Urban J., 1998, Asset Pricing in Production Economies, Journal of Monetary Economics, Vol. 41 (2)., April, 257-75.

[16] Lewis, Karen K., 1996, Consumption, Stock Returns, and the Gains from International Risk- Sharing, NBER Working Paper: 5410.

[17] Lucas, Robert, E. Jr., 1987, Models of Business Cycles, Basil Blackwell, New York.

[18] Mehra, Rajnish and Prescott, Edward C., 1985, The Equity Premium: A Puzzle, Journal of Monetary Economics, v15(2), 145-161.

[19] Obstfeld, Maurice, 1994, Evaluating Risky Consumption Paths: The Role of Intertemporal Substitutability, European Economic Review 38, 1471-1486.

[20] Otrok, Christopher, 1998, On Measuring The Welfare Cost of Business Cycles, University of Virginia.

[21] Rietz, Thomas A., 1988, The Equity Risk Premium: A Solution, Journal of Monetary Economics, v22 n1 July, 117-31.

[22] Shiller, Robert J., 1998, Annual Data on US Stock Market Prices, Dividends, Earnings, 1871-Present, with Associated Interest Rate, Price Level and Consumption Data, http://www.econ.yale.edu/ shiller/chapt26.html.

[23] Tallarini, Thomas D. Jr., 2000, Risk-Sensitive Real Business Cycles, Journal of Monetary Economics, 45, 507-532.

[24] Van Wincoop, Eric, 1999, How Big Are Potential Welfare Gains from International Risksharing?, Journal of International Economics, Vol. 47 (1), 109-35. 


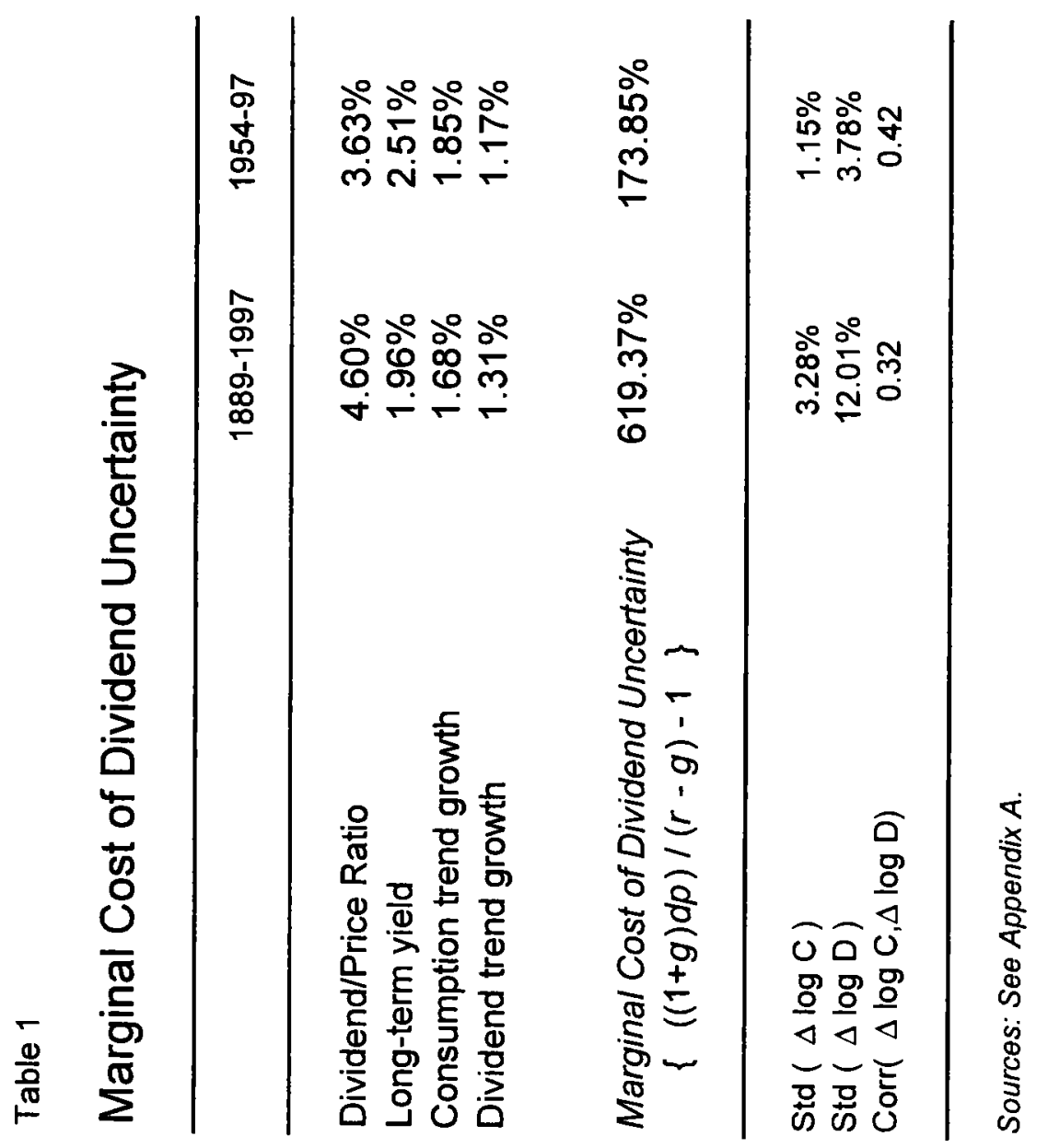


Table 2A

\section{Marginal Cost of Consumption Fluctuations (1889-1997)}

Moments the kernel fits:

Coefficients on states:

\begin{tabular}{|c|c|c|c|c|c|c|}
\hline & $\mathrm{c}$ & d & trm & $d / p$ & vwr & $\beta$ \\
\hline 1. $E(D / P), E(V W R-R f), Y(1), Y(13)$, & -9.31 & -1.56 & 8.05 & & & 0.90 \\
\hline 2. $E(R d / R f), E(V W R-R f), Y(1), Y(13)$, & -9.21 & -1.58 & 8.19 & & & 0.90 \\
\hline 3. $E(D / P), E(R d / R f), Y(1), Y(13)$ & -9.08 & -1.59 & 8.31 & & & 0.90 \\
\hline 4. $E(D / P), E(V W R-R f), Y(1), Y(13)$, & -4.04 & -2.37 & & 9.64 & & 0.91 \\
\hline 5. $E(R d / R f), E(V W R-R f), Y(1), Y(13)$, & -0.19 & -3.31 & & 10.09 & & 0.90 \\
\hline 6. $E(D / P), E(R d / R f), Y(1), Y(13)$, & -4.31 & -2.57 & & 10.22 & & 0.90 \\
\hline 7. $E(D / P), E(R d / R f), Y(1), Y(13), E(V W R-R f)$ & -9.60 & -1.53 & 8.19 & -0.49 & & 0.90 \\
\hline 8. $E(D / P), E(V W R-R f), Y(1), Y(13)$, & -6.53 & -1.93 & & & -0.53 & 0.90 \\
\hline 9. $E(R d / R f), E(V W R-R f), Y(1), Y(13)$, & 0.06 & -3.30 & & & -0.70 & 0.89 \\
\hline 10. $E(D / P), E(R d / R f), Y(1), Y(13)$ & -1.00 & -2.88 & & & -0.69 & 0.91 \\
\hline 11. $E(D / P), E(V W R-R f), Y(1), Y(13)$, & -7.18 & -1.98 & & & -0.40 & 0.90 \\
\hline 12. $E(R d / R f), E(V W R-R f), Y(1), Y(13)$, & -0.69 & -3.24 & & & -0.63 & 0.89 \\
\hline 13. $E(D / P), E(R d / R f), Y(1), Y(13),[O l+E(V W R-R f)]$ & -5.36 & -2.25 & & & -0.49 & 0.91 \\
\hline 14. $E(D / P), E(R d / R f), Y(1), Y(13), E(V W R-R f)$ & 0.06 & -2.16 & & 44.74 & 1.66 & 0.93 \\
\hline 15. $E(D / P), E(R d / R f), Y(1), Y(13), E(V W R-R f)$ & -13.44 & -1.04 & 8.61 & & 0.41 & 0.88 \\
\hline
\end{tabular}

Standard deviation of consumption deviations from trend

\begin{tabular}{|c|c|c|c|}
\hline Moments & $\begin{array}{l}\text { Dividend/price ratio: } E(D / P)=0.046 \\
\text { Equity premium: } E(W W R-R f)=0.0677 \\
\text { Multiplicative equity premium: } E(R d / R f-1)=0.0698 \\
\text { Riskless rate: } E(\log Y(1))=0.0108 \\
\text { Yield spread: } E(\log Y(13)-\log Y(1))=0.0059\end{array}$ & States & $\begin{array}{l}c=\text { consumption } \\
d=\text { dividends } \\
\text { trm=long-short term spread } \\
d / p=\text { dividend-price ratio } \\
v w r=\text { realized value weighted stock return }\end{array}$ \\
\hline
\end{tabular}


Table 2A, continued

\section{Marginal Cost of Consumption Fluctuations (1889-1997)}

\begin{tabular}{|c|c|c|c|c|c|c|c|c|c|}
\hline $\begin{array}{c}\text { Low pass fi } \\
8 \text { years }\end{array}$ & s 12 years & Marginal c & 20 years & $\begin{array}{c}\begin{array}{c}\text { Other Filters } \\
\text { infinite }\end{array} \\
\end{array}$ & geometric & linear & $\begin{array}{l}\text { Benefit } \\
\text { of } 1 \% \\
\text { growth }\end{array}$ & $\begin{array}{c}\text { Duration } \\
\text { Sonsumption } \\
\text { Equity }\end{array}$ & Fit \\
\hline $0.43 \%$ & $0.79 \%$ & $0.89 \%$ & $1.06 \%$ & $353.26 \%$ & $1.01 \%$ & $1.85 \%$ & $227.60 \%$ & 70.19 & 0 \\
\hline $0.43 \%$ & $0.79 \%$ & $0.89 \%$ & $1.05 \%$ & $347.07 \%$ & $0.94 \%$ & $1.73 \%$ & $227.26 \%$ & 70.16 & 0 \\
\hline $0.42 \%$ & $0.78 \%$ & $0.88 \%$ & $1.05 \%$ & $341.72 \%$ & $0.99 \%$ & $1.83 \%$ & $230.56 \%$ & 70.47 & 0 \\
\hline $0.32 \%$ & $0.60 \%$ & $0.67 \%$ & $0.69 \%$ & $543.28 \%$ & $0.72 \%$ & $1.41 \%$ & $1560.88 \%$ & 98.90 & 0 \\
\hline $0.24 \%$ & $0.48 \%$ & $0.54 \%$ & $0.64 \%$ & $604.27 \%$ & $0.61 \%$ & $1.15 \%$ & $780.47 \%$ & 115.55 & 0 \\
\hline $0.35 \%$ & $0.65 \%$ & $0.73 \%$ & $0.86 \%$ & $1956.35 \%$ & $0.82 \%$ & $1.52 \%$ & $4141.52 \%$ & 109.09 & 1.43E-05 \\
\hline $0.43 \%$ & $0.80 \%$ & $0.90 \%$ & $1.07 \%$ & $362.85 \%$ & $1.02 \%$ & $1.88 \%$ & $221.43 \%$ & 69.59 & 0 \\
\hline $0.40 \%$ & $0.73 \%$ & $0.80 \%$ & $0.93 \%$ & $2862.13 \%$ & $0.93 \%$ & $1.68 \%$ & $1344.64 \%$ & 97.20 & 0 \\
\hline $0.26 \%$ & $0.49 \%$ & $0.54 \%$ & $0.63 \%$ & $1263.46 \%$ & $0.63 \%$ & $1.16 \%$ & $16561.54 \%$ & 123.50 & 0 \\
\hline $0.27 \%$ & $0.51 \%$ & $0.55 \%$ & $0.64 \%$ & $6151.07 \%$ & $0.64 \%$ & $1.19 \%$ & $154985.17 \%$ & 151.48 & 2.62E-05 \\
\hline $0.40 \%$ & $0.71 \%$ & $0.79 \%$ & $0.93 \%$ & $502.50 \%$ & $0.90 \%$ & $1.65 \%$ & $571.11 \%$ & 86.50 & 0 \\
\hline $0.26 \%$ & $0.49 \%$ & $0.55 \%$ & $0.65 \%$ & $480.22 \%$ & $0.63 \%$ & $1.16 \%$ & $8664.40 \%$ & 116.65 & 0 \\
\hline $0.36 \%$ & $0.64 \%$ & $0.72 \%$ & $0.84 \%$ & $605.66 \%$ & $0.82 \%$ & $1.49 \%$ & $1498.99 \%$ & 98.47 & 2.72E-06 \\
\hline $0.20 \%$ & $0.43 \%$ & $0.54 \%$ & $0.68 \%$ & $39.70 \%$ & $0.49 \%$ & $0.95 \%$ & $109.37 \%$ & 52.82 & 0 \\
\hline $0.50 \%$ & $0.92 \%$ & $1.06 \%$ & $1.27 \%$ & $206.03 \%$ & $1.18 \%$ & $2.17 \%$ & $110.82 \%$ & 53.17 & 5.78E-08 \\
\hline $1.35 \%$ & $1.68 \%$ & $2.02 \%$ & $2.36 \%$ & & $1.80 \%$ & $2.92 \%$ & & & \\
\hline
\end{tabular}

$$
\begin{array}{lll}
\text { Forecasting } & 1 .-7 . & c, d, t m, d / p \\
\text { variables } & 8 .-10 ., 14 . & c, d, d p, v w r \\
& 11 .-13,15 . & c, d, t m, v w r
\end{array}
$$

Fit stands for the sum of squared residuals from data moments 
Table 2B

\section{Marginal Cost of Consumption Fluctuations (1954-1997)}

Moments the kernel fits:

Coefficients on states:

\begin{tabular}{l|l}
\hline &
\end{tabular}

1. $E(D / P), E(V W R-R f), Y(1), Y(13)$,

c

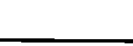

2. $E(R d / R f), E(V W R-R f), Y(1), Y(13)$,

3. $E(D / P), E(R d / R f), Y(1), Y(13),[O I+E(V W R-R f)]$

$-28.64 \quad-2.82 \quad 23.95$

$\mathrm{d} / \mathrm{p} \quad \mathrm{wwr}$ $\beta$

$-19.30$

6.49

41.62

$-12.61$

$-2.40$

20.16

0.85

(n)

4. $E(D / P), E(V W R-R f), Y(1), Y(13)$,

$-10.94 \quad-4.40$

53.35

0.92

5. $E(R d / R f), E(W W R-R f), Y(1), Y(13)$, [no trm, OI + E(VWR-Rf)]

$-15.96$

$-2.93$

41.14

0.91

6. $E(D / P), E(R d / R f), Y(1), Y(13)$, [no trm state]

$-7.19 \quad-3.22$

41.53

0.93

7. $E(D / P), E(R d / R f), Y(1), Y(13), E(V W R-R f)$

no fit

8. $E(D / P), E(V W R-R f), Y(1), Y(13)$,

9. $E(R d / R f), E(W W R-R f), Y(1), Y(13)$,

10. $E(D / P), E(R d / R f), Y(1), Y(13), \quad(1947-1997)$

11. $E(D / P), E(V W R-R f), Y(1), Y(13),(1947-1997)$

12. $E(R d / R f), E(V W R-R f), Y(1), Y(13),[$ no trm, $O I+E(D / P)]$

$-11.85$

$-4.28$

$-2.02$

0.89

$-36.53 \quad-5.93$

$-2.13$

0.75

$-23.70-5.20$

$-1.88$

0.84

$\begin{array}{ll}-8.62 & -4.99\end{array}$

$-2.04$

0.92

$-0.95-4.50$

$-1.90$

0.92

13. $E(D / P), E(R d / R f), Y(1), Y(13)$, [no trm state]

$-0.88$

$-3.84$

$-1.72$

0.93

14. $E(D / P), E(R d / R f), Y(1), Y(13), E(V W R-R f)$

$-40.57$

5.40

94.47

1.16

0.71

15. $E(D / P), E(R d / R f), Y(1), Y(13), E(W W R-R f)$

no fit

Standard deviation of consumption deviations from trend

Moments Dividend/price ratio: $E(D / P)=0.0363$

Equity premium: $E(V W R-R f)=0.0628$

Multiplicative equity premium: $E(R d / R f-1)=0.0613$

Riskless rate: $E(\log Y(1))=0.0121$

Yield spread: $E(\log Y(13)-\log Y(1))=0.0123$
States $\quad c=$ consumption

$d=$ dividends

trm=long-short term spread

$d / p=$ dividend-price ratio

$v w r=$ realized value weighted stock return 
Table 2B, continued

\section{Marginal Cost of Consumption Fluctuations (1954-1997)}

\begin{tabular}{|c|c|c|c|c|c|c|c|c|c|}
\hline \multicolumn{2}{|c|}{ Low pass filters } & \multicolumn{2}{|c|}{ Marginal cost: } & \multirow{2}{*}{$\begin{array}{c}\text { Other filters } \\
\text { inf }\end{array}$} & \multirow[b]{2}{*}{ geometric } & \multirow[b]{2}{*}{ linear } & \multirow{2}{*}{$\begin{array}{l}\text { Benefit } \\
\text { of } 1 \% \\
\text { growth }\end{array}$} & \multirow{2}{*}{$\begin{array}{c}\text { Duration } \\
\text { Consumption } \\
\text { Equity } \\
\end{array}$} & \multirow[b]{2}{*}{ Fit } \\
\hline 8 years & 12 years & 16 years & 20 years & & & & & & \\
\hline $0.22 \%$ & $0.42 \%$ & $0.51 \%$ & $0.62 \%$ & $22.33 \%$ & $0.51 \%$ & $0.96 \%$ & $6341.00 \%$ & 39.28 & 0 \\
\hline $0.15 \%$ & $0.31 \%$ & $0.35 \%$ & $0.41 \%$ & $302.10 \%$ & $0.37 \%$ & $0.70 \%$ & $312531307.00 \%$ & 211.65 & 0 \\
\hline $0.11 \%$ & $0.22 \%$ & $0.27 \%$ & $0.33 \%$ & $11.54 \%$ & $0.26 \%$ & $0.48 \%$ & $103.93 \%$ & 51.5078 & $6.38 \mathrm{E}-05$ \\
\hline $0.14 \%$ & $0.22 \%$ & $0.24 \%$ & $0.26 \%$ & $17.86 \%$ & $0.24 \%$ & $0.41 \%$ & $203949.00 \%$ & 102.06 & 0 \\
\hline $0.15 \%$ & $0.27 \%$ & $0.32 \%$ & $0.39 \%$ & $32.77 \%$ & ,0036 & $0.66 \%$ & $144.60 \%$ & 59.76 & $6.63 \mathrm{E}-06$ \\
\hline $0.09 \%$ & $0.16 \%$ & $0.18 \%$ & $0.23 \%$ & $18.72 \%$ & $0.21 \%$ & $0.38 \%$ & $217.64 \%$ & 69.32 & $1.02 \mathrm{E}-06$ \\
\hline $0.18 \%$ & $0.34 \%$ & $0.36 \%$ & $0.41 \%$ & $115570.55 \%$ & $0.44 \%$ & $0.81 \%$ & $290802426.00 \%$ & 508.09 & 0 \\
\hline $0.32 \%$ & $0.57 \%$ & $0.60 \%$ & $0.69 \%$ & $1156.26 \%$ & $0.72 \%$ & $1.29 \%$ & $2530.00 \%$ & 103.28 & 0 \\
\hline $0.21 \%$ & $0.34 \%$ & $0.37 \%$ & $0.42 \%$ & $54.54 \%$ & $0.43 \%$ & $0.75 \%$ & $23430.00 \%$ & 70.94 & $7.66 \mathrm{E}-07$ \\
\hline $0.14 \%$ & $0.21 \%$ & $0.24 \%$ & $0.28 \%$ & $18.32 \%$ & $0.25 \%$ & $0.43 \%$ & $2965341.00 \%$ & 129.94 & 0 \\
\hline $0.07 \%$ & $0.12 \%$ & $0.12 \%$ & $0.14 \%$ & $248.02 \%$ & $0.16 \%$ & $0.27 \%$ & $141029900.00 \%$ & 419.44 & 8.78E-06 \\
\hline $0.07 \%$ & $0.10 \%$ & $0.11 \%$ & $0.13 \%$ & $38.50 \%$ & $0.14 \%$ & $0.23 \%$ & $335717.05 \%$ & 163.47 & 7.95E-06 \\
\hline $0.42 \%$ & $0.73 \%$ & $0.90 \%$ & $1.01 \%$ & $35.57 \%$ & $0.97 \%$ & $1.79 \%$ & $3149.00 \%$ & 24.44 & 0 \\
\hline $0.55 \%$ & $0.69 \%$ & $0.67 \%$ & $0.70 \%$ & & $0.73 \%$ & $1.21 \%$ & & & \\
\hline
\end{tabular}

$\begin{array}{lll}\text { Forecasting } & 1 .-7 . & c, d, t r m, d / p \\ \text { variables } & 8 .-10 ., 14 . & c, d, d p, w w r \\ & 11 .-13,15 . & c, d, t r m, w w r\end{array}$

Fit stands for the sum of squared residuals from data moments 
Table 2C

Marginal Cost of Consumption Fluctuations: Dividends and Consumption Cointegrated (1889-1997)

\begin{tabular}{|c|c|c|c|c|c|c|}
\hline Moments the kernel fits: & $\begin{array}{c}\text { Coefficien } \\
\text { c }\end{array}$ & $\begin{array}{c}\text { states: } \\
\quad \mathrm{d}\end{array}$ & $\mathrm{trm}$ & $d / p$ & vwr & $\beta$ \\
\hline 1. $E(D / P), E(V W R-R f), Y(1), Y(13),[O I+E(R d / R f)$ & -11.55 & -1.40 & -8.19 & & & 0.90 \\
\hline 2. $E(R d / R f), E(V W R-R f), Y(1), Y(13),[O I+E(D / P)]$ & -11.55 & -1.40 & -8.19 & & & 0.90 \\
\hline 3. $E(D / P), E(R d / R f), Y(1), Y(13),[O I+E(W V R-R f)]$ & -11.55 & -1.40 & -8.19 & & & 0.90 \\
\hline 4. $E(D / P), E(V W R-R f), Y(1), Y(13)$, & -17.93 & -3.47 & & -32.32 & & 0.81 \\
\hline 5. $E(R d / R f), E(V W R-R f), Y(1), Y(13),[O I+E(D / P)]$ & -16.40 & -1.18 & & -10.29 & & 0.86 \\
\hline 6. $E(D / P), E(R d / R f), Y(1), Y(13),[O I+E(W V R-R f)]$ & -16.40 & -1.18 & & -10.29 & & 0.86 \\
\hline 7. $E(D / P), E(R d / R f), Y(1), Y(13), E(V W R-R f)$ & no fit & & & & & \\
\hline 8. $E(D / P), E(V W R-R f), Y(1), Y(13)$, & -10.28 & -3.54 & & & 1.05 & 0.88 \\
\hline 9. $E(R d / R f), E(V W R-R f), Y(1), Y(13)$, & -20.06 & 0.23 & & & 0.41 & 0.83 \\
\hline 10. $E(D / P), E(R d / R f), Y(1), Y(13),[O I+E(W V R-R f)]$ & -8.80 & -2.44 & & & 0.40 & 0.91 \\
\hline 11. $E(D / P), E(V W R-R f), Y(1), Y(13)$, [no trm state] & -21.79 & -3.54 & & & 3.17 & 0.75 \\
\hline 12. $E(R d / R f), E(V W R-R f), Y(1), Y(13),[$ no trm state] & -21.95 & 0.03 & & & 1.03 & 0.81 \\
\hline 13. $E(D / P), E(R d / R f), Y(1), Y(13),[O I+E(V W R-R f)]$ & -10.94 & -2.20 & & & 0.95 & 0.90 \\
\hline 14. $E(D / P), E(R d / R f), Y(1), Y(13), E(V W R-R f)$ & -10.50 & 1.79 & & 75.51 & 3.09 & 0.90 \\
\hline 15. $E(D / P), E(R d / R f), Y(1), Y(13), E(V W R-R f)$ & no fit & & & & & \\
\hline
\end{tabular}

Standard deviation of consumption deviations from trend

\begin{tabular}{|c|c|c|c|}
\hline Moments & $\begin{array}{l}\text { Dividend/price ratio: } E(D / P)=0.046 \\
\text { Equity premium: } E(V W R-R f)=0.0677 \\
\text { Multiplicative equity premium: } E(R d / R f-1)=0.0698 \\
\text { Riskless rate: } E(\log Y(1))=0.0108 \\
\text { Yield spread: } E(\log Y(13)-\log Y(1))=0.0059\end{array}$ & States & $\begin{array}{l}c=\text { consumption } \\
d=\text { dividends } \\
\text { trm=long-short term spread } \\
d / p=\text { dividend-price ratio } \\
v w r=\text { realized value weighted stock retum }\end{array}$ \\
\hline
\end{tabular}


Table 2C, continued

Marginal Cost of Consumption Fluctuations: Dividends and

Consumption Cointegrated (1889-1997)

\begin{tabular}{|c|c|c|c|c|c|c|c|c|c|}
\hline $\begin{array}{c}\text { Low pass filters } \\
8 \text { years } \\
\end{array}$ & 12years & 16 years & 20 years & $\begin{array}{c}\text { Other filters } \\
\text { infinite }\end{array}$ & geometric & linear & $\begin{array}{l}\text { Benefit } \\
\text { of } 1 \% \\
\text { growth }\end{array}$ & $\begin{array}{c}\text { Duration } \\
\text { Consumption } \\
\text { Equity } \\
\end{array}$ & Fit \\
\hline $0.36 \%$ & $0.66 \%$ & $0.78 \%$ & $0.96 \%$ & $47.68 \%$ & $0.88 \%$ & $1.60 \%$ & $45.98 \%$ & 31.74 & $2.19 E-04$ \\
\hline $0.36 \%$ & $0.66 \%$ & $0.78 \%$ & $0.96 \%$ & $47.68 \%$ & $0.88 \%$ & $1.60 \%$ & $45.98 \%$ & 31.74 & 2.19E-04 \\
\hline $0.36 \%$ & $0.66 \%$ & $0.78 \%$ & $0.96 \%$ & $47.68 \%$ & $0.88 \%$ & $1.60 \%$ & $45.98 \%$ & 31.74 & $2.19 E-04$ \\
\hline $0.37 \%$ & $0.81 \%$ & $0.98 \%$ & $1.25 \%$ & $196.30 \%$ & $1.20 \%$ & $2.29 \%$ & $64.22 \%$ & 39.12 & $0.00 E+00$ \\
\hline $0.49 \%$ & $0.90 \%$ & $1.07 \%$ & $1.31 \%$ & $88.99 \%$ & $1.19 \%$ & $2.20 \%$ & $51.31 \%$ & 34.09 & $1.91 E-04$ \\
\hline $0.49 \%$ & $0.90 \%$ & $1.07 \%$ & $1.31 \%$ & $88.99 \%$ & $1.19 \%$ & $2.20 \%$ & $51.31 \%$ & 34.09 & $1.91 E-04$ \\
\hline $0.23 \%$ & $0.56 \%$ & $0.71 \%$ & $0.93 \%$ & $55.17 \%$ & $0.84 \%$ & $1.65 \%$ & $40.02 \%$ & 28.94 & 0 \\
\hline $0.67 \%$ & $1.22 \%$ & $1.37 \%$ & $1.63 \%$ & $367250.00 \%$ & $1.59 \%$ & $2.90 \%$ & $231.55 \%$ & 70.40 & 0 \\
\hline $0.27 \%$ & $0.55 \%$ & $0.68 \%$ & $0.86 \%$ & $45.20 \%$ & $0.75 \%$ & $1.43 \%$ & $44.36 \%$ & 31.06 & $1.66 \mathrm{E}-04$ \\
\hline $0.43 \%$ & $1.05 \%$ & $1.23 \%$ & $1.53 \%$ & $560455113.38 \%$ & $1.42 \%$ & 0.0278 & $214.25 \%$ & 66.59 & 0 \\
\hline $0.66 \%$ & $1.27 \%$ & $1.39 \%$ & $1.63 \%$ & $1.17 \mathrm{E}+12$ & $1.64 \%$ & $3.02 \%$ & $5.05 E+04$ & 224.70 & 5.37E-05 \\
\hline $0.28 \%$ & $0.58 \%$ & $0.70 \%$ & $0.87 \%$ & $61.68 \%$ & $0.77 \%$ & $1.46 \%$ & $55.90 \%$ & 35.95 & 2.77E-04 \\
\hline $0.51 \%$ & $0.86 \%$ & $1.08 \%$ & $1.32 \%$ & $19.94 \%$ & $1.07 \%$ & $1.96 \%$ & $22.74 \%$ & 18.86 & 0 \\
\hline $1.35 \%$ & $1.68 \%$ & $2.02 \%$ & $2.36 \%$ & & $1.80 \%$ & $2.92 \%$ & & & \\
\hline
\end{tabular}

$\begin{array}{lll}\text { Forecasting } & 1 .-7 . & c, d, t r m, d p \\ \text { variables } & 8 .-10 ., 14 . & c, d, d p, v w r \\ & 11 .-13,15 . & c, d, t m, v w r\end{array}$

Fit stands for the sum of squared residuals from data moments 
Table 2D

Marginal Cost of Consumption Fluctuations: Dividends and Consumption Cointegrated (1954-1997)

\begin{tabular}{|c|c|c|c|c|c|c|}
\hline Moments the kernel fits: & $\begin{array}{c}\text { Coefficier } \\
\mathrm{c}\end{array}$ & $\begin{array}{c}\text { on stat } \\
\text { d }\end{array}$ & $\operatorname{trm}$ & $d / p$ & nwr & $\beta$ \\
\hline 1. $E(D / P), E(V W R-R f), Y(1), Y(13)$, & -17.62 & -1.68 & 31.10 & & & 0.91 \\
\hline 2. $E(R d / R f), E(V W R-R f), Y(1), Y(13),[n o d / p, O i+E(D / P)]$ & 6.54 & -4.95 & 22.05 & & & 0.95 \\
\hline 3. $E(D / P), E(R d / R f), Y(1), Y(13),[n o d / p, O I+E(R d / R f)]$ & 6.54 & -4.95 & 22.05 & & & 0.95 \\
\hline 4. $E(D / P), E(V W R-R f), Y(1), Y(13)$, & -5.92 & -2.39 & & 64.75 & & 0.94 \\
\hline 5. $E(R d / R f), E(V W R-R f), Y(1), Y(13)$, & -9.89 & -0.52 & & 60.82 & & 0.95 \\
\hline 6. $E(D / P), E(R d / R f), Y(1), Y(13)$ & 3.47 & -3.07 & & 57.59 & & 0.95 \\
\hline 7. $E(D / P), E(R d / R f), Y(1), Y(13), E(V W R-R f)$. & 0.83 & -2.97 & 1.72 & 56.73 & & 0.95 \\
\hline 8. $E(D / P), E(V W R-R f), Y(1), Y(13)$, & -13.95 & -4.61 & & & -1.89 & 0.88 \\
\hline 9. $E(R d / R f), E(V W R-R f), Y(1), Y(13)$, & -18.46 & -2.94 & & & -1.97 & 0.88 \\
\hline 10. $E(D / P), E(R d / R f), Y(1), Y(13)$, & -6.54 & -4.50 & & & -1.46 & 0.93 \\
\hline 11. $E(D / P), E(V W R-R f), Y(1), Y(13)$, & -13.34 & -4.58 & & & -1.92 & 0.91 \\
\hline 12. $E(R d / R f), E(V W R-R f), Y(1), Y(13),[O I+E(D / P)]$ & -10.18 & -4.45 & & & -1.64 & 0.91 \\
\hline 13. $E(D / P), E(R d / R f), Y(1), Y(13),[O I+E(V W R-R f)]$ & -10.18 & -4.45 & & & -1.64 & 0.91 \\
\hline 14. $E(D / P), E(R d / R f), Y(1), Y(13), E(V W R-R f)$, & -4.73 & -4.84 & -1.61 & & -2.78 & 0.94 \\
\hline 15. $E(D / P), E(R d / R f), Y(1), Y(13), E(V W R-R f)$, & -12.87 & -3.42 & & 31.18 & -0.70 & 0.90 \\
\hline
\end{tabular}

Standard deviations of consumption deviations from trend

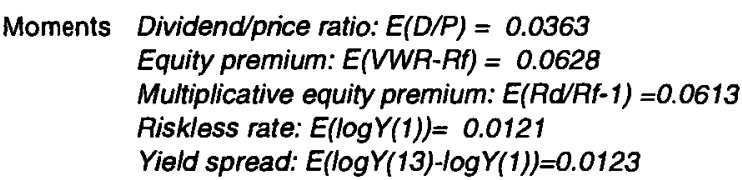

States $\quad c=$ consumption
$d=$ dividends
$t m=$ long-short tem spread
$d / p=$ dividend-price ratio
$w w=$ realized value weighted stock return


Table 2D, continued

Marginal Cost of Consumption Fluctuations: Dividends and

Consumption Cointegrated (1954-1997)

\begin{tabular}{|c|c|c|c|c|c|c|c|c|c|}
\hline $\begin{array}{l}\text { Low pass } \\
\mathbf{8} \text { years }\end{array}$ & $\begin{array}{l}\text { Iters } \\
12 \text { years }\end{array}$ & Marginal c & 20 years & $\begin{array}{c}\text { Other filters } \\
\text { inf }\end{array}$ & geometric & linear & $\begin{array}{l}\text { Benefit } \\
\text { of } 1 \% \\
\text { growth }\end{array}$ & $\begin{array}{c}\text { Duration } \\
\text { Consumption } \\
\text { Equity }\end{array}$ & Fit \\
\hline $0.22 \%$ & $0.42 \%$ & $0.52 \%$ & $0.66 \%$ & $25.55 \%$ & $0.53 \%$ & $1.00 \%$ & $56.39 \%$ & 36.66 & 0 \\
\hline $0.11 \%$ & $0.19 \%$ & $0.30 \%$ & $0.40 \%$ & $0.26 \%$ & $0.22 \%$ & $0.42 \%$ & $52.12 \%$ & 34.70 & $3.28 \mathrm{E}-05$ \\
\hline $0.11 \%$ & $0.19 \%$ & $0.30 \%$ & $0.40 \%$ & $0.26 \%$ & $0.22 \%$ & $0.42 \%$ & $52.12 \%$ & 34.70 & 3.28E-05 \\
\hline $0.19 \%$ & $0.28 \%$ & $0.34 \%$ & $0.42 \%$ & $8.07 \%$ & $0.33 \%$ & $0.58 \%$ & $45.07 \%$ & 31.68 & 0 \\
\hline $0.17 \%$ & $0.25 \%$ & $0.28 \%$ & $0.33 \%$ & $21.06 \%$ & $0.31 \%$ & $0.53 \%$ & $137.86 \%$ & 58.81 & 2.27E-06 \\
\hline $0.12 \%$ & $0.16 \%$ & $0.21 \%$ & $0.27 \%$ & $0.16 \%$ & $0.17 \%$ & $0.29 \%$ & $46.35 \%$ & 32.23 & 5.97E-07 \\
\hline $0.140 \%$ & $0.190 \%$ & $0.260 \%$ & $0.320 \%$ & $2.41 \%$ & $0.23 \%$ & $0.38 \%$ & $45.34 \%$ & 31.77 & 3.54E-06 \\
\hline $0.33 \%$ & $0.59 \%$ & $0.71 \%$ & $0.87 \%$ & $48.16 \%$ & $0.77 \%$ & $1.40 \%$ & $57.17 \%$ & 36.97 & 0 \\
\hline $0.35 \%$ & $0.67 \%$ & $0.71 \%$ & $0.82 \%$ & $462789900.00 \%$ & $0.86 \%$ & $0.16 \%$ & $89197900.00 \%$ & 375.47 & 0 \\
\hline $0.24 \%$ & $0.41 \%$ & $0.51 \%$ & $0.65 \%$ & $20.54 \%$ & $0.53 \%$ & $0.96 \%$ & $46.22 \%$ & 32.20 & 0 \\
\hline $0.29 \%$ & $0.48 \%$ & $0.58 \%$ & $0.70 \%$ & $20.53 \%$ & $0.59 \%$ & $1.05 \%$ & $49.25 \%$ & 33.60 & 0 \\
\hline $0.28 \%$ & $0.49 \%$ & $0.60 \%$ & $0.75 \%$ & $31.13 \%$ & $0.64 \%$ & $1.15 \%$ & $50.85 \%$ & 34.31 & 4.55E-06 \\
\hline $0.28 \%$ & $0.49 \%$ & $0.60 \%$ & $0.75 \%$ & $31.13 \%$ & $0.64 \%$ & $1.15 \%$ & $50.85 \%$ & 34.31 & 4.55E-06 \\
\hline $0.22 \%$ & $0.34 \%$ & $0.43 \%$ & $0.53 \%$ & $9.80 \%$ & $0.41 \%$ & $0.73 \%$ & $45.30 \%$ & 31.72 & 1.33E-05 \\
\hline $0.28 \%$ & $0.50 \%$ & $0.60 \%$ & $0.75 \%$ & $38.05 \%$ & $0.65 \%$ & $1.19 \%$ & $54.36 \%$ & 35.90 & 5.53E-06 \\
\hline $0.55 \%$ & $0.69 \%$ & $0.67 \%$ & $0.70 \%$ & & $0.73 \%$ & $1.21 \%$ & & & \\
\hline
\end{tabular}

$\begin{array}{lll}\text { Forecasting } & 1 .-7 . & c, d, t m, d / p \\ \text { variables } & 8 .-10 ., 14 . & c, d, d p, v w r \\ & 11,-13,15 . & c, d, t m, v w r\end{array}$

Fit stands for sum of squared residuals from data moments 


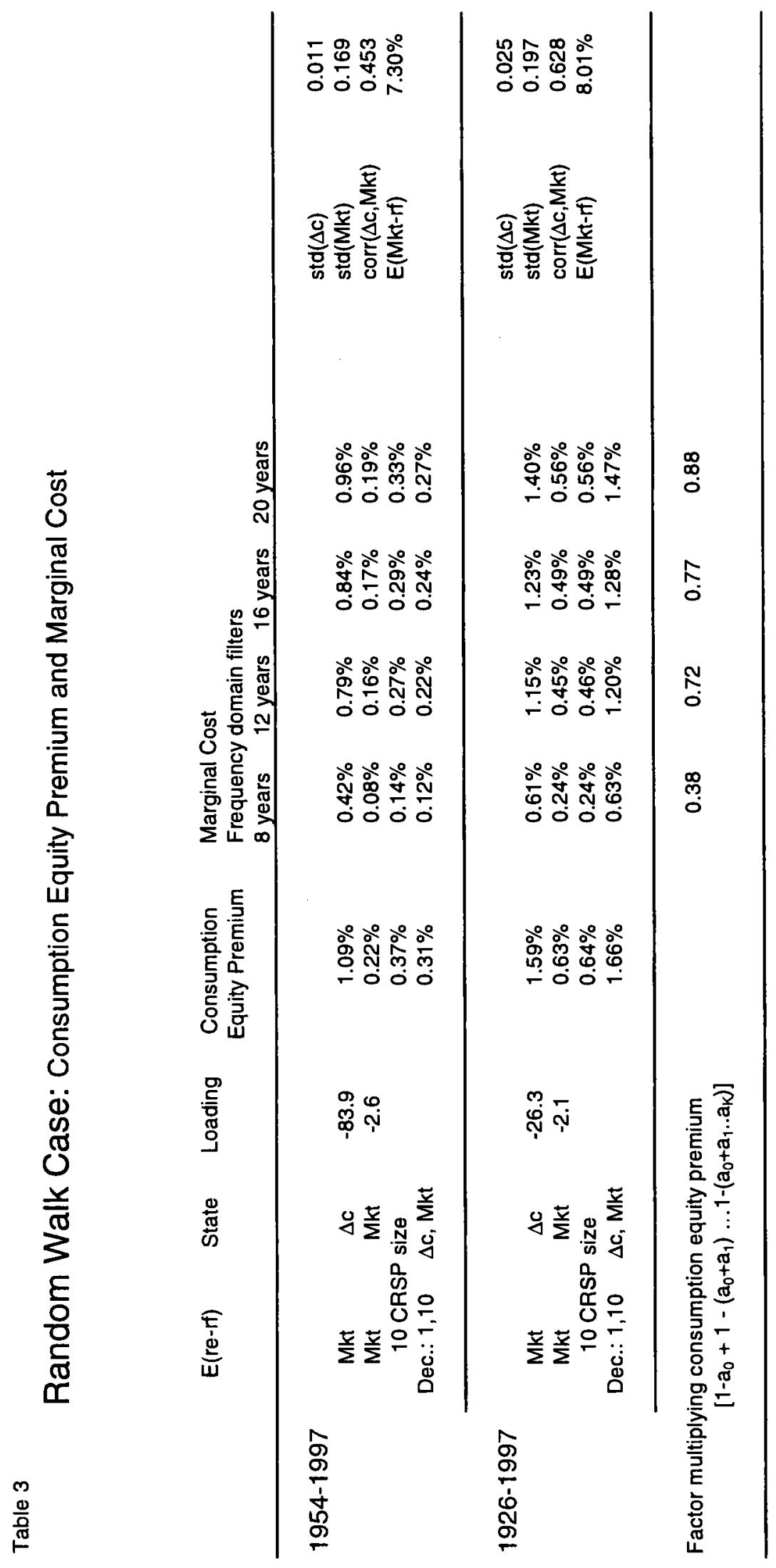


Figure 1: $\quad$ Marginal and Total Cost Functions
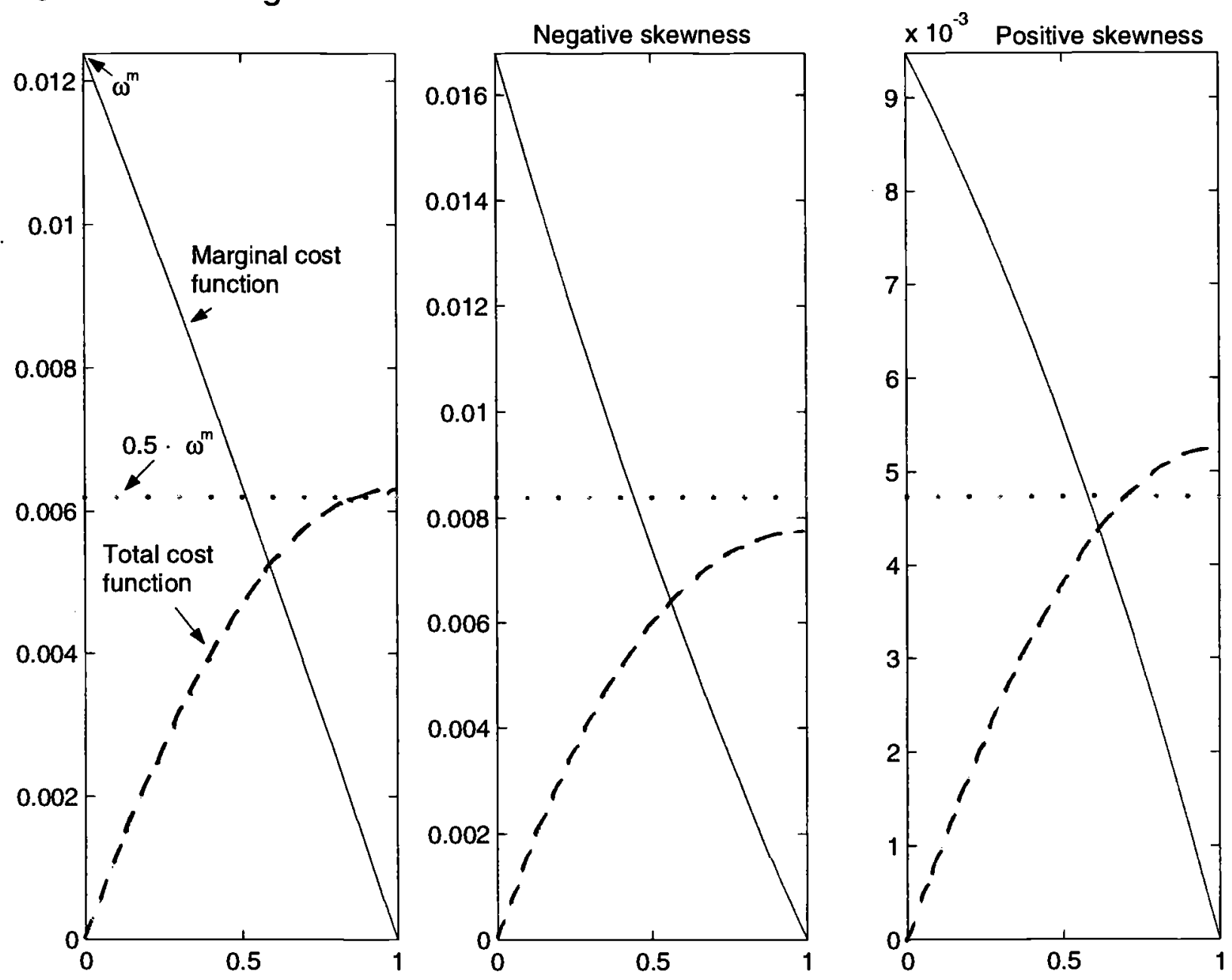

$\alpha$ : share of volatility removed 
Figure 2 Transfer functions for low-pass filters, 8 year cutoff (15 lags)
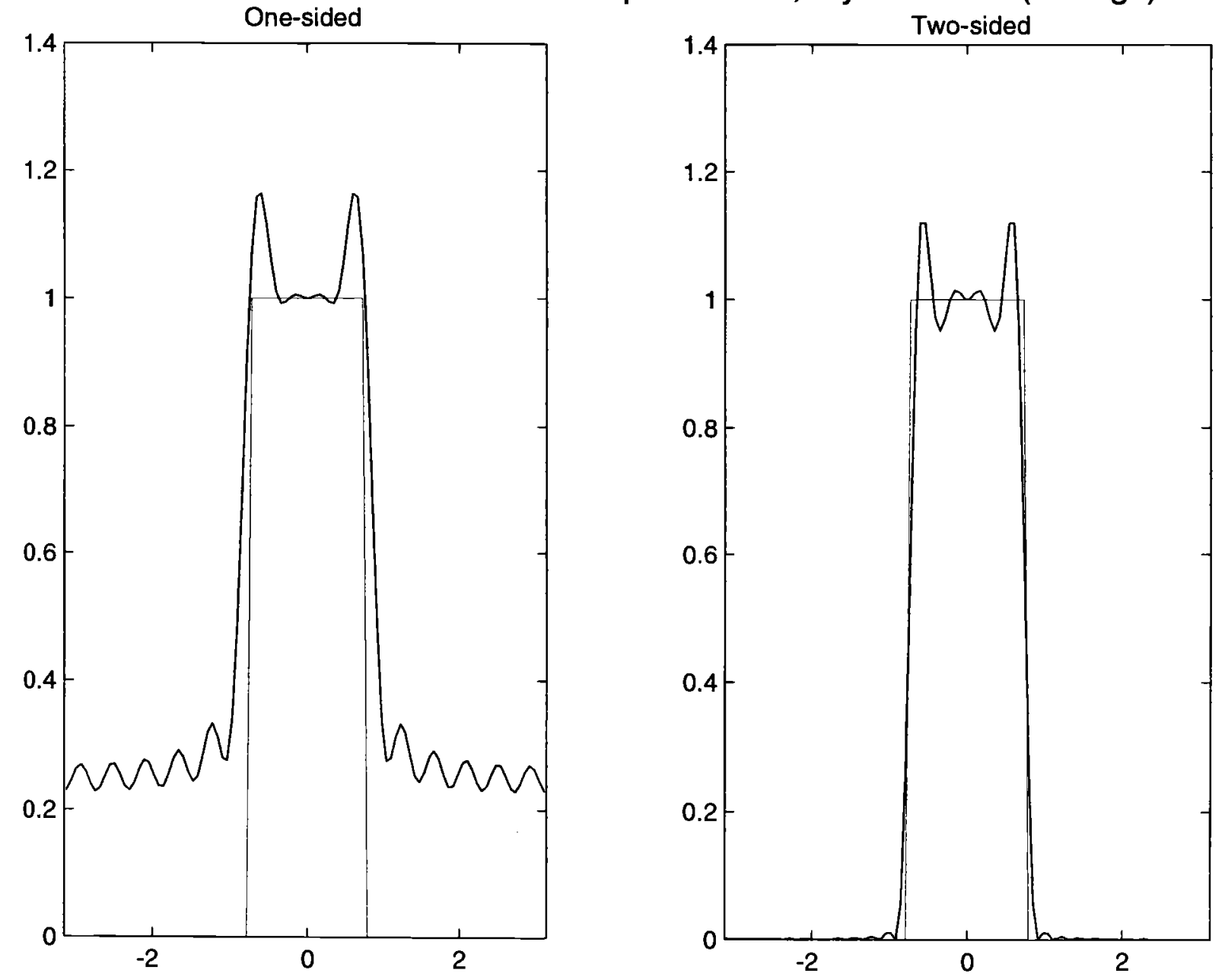
Figure 3A Consumption Deviations from Trend
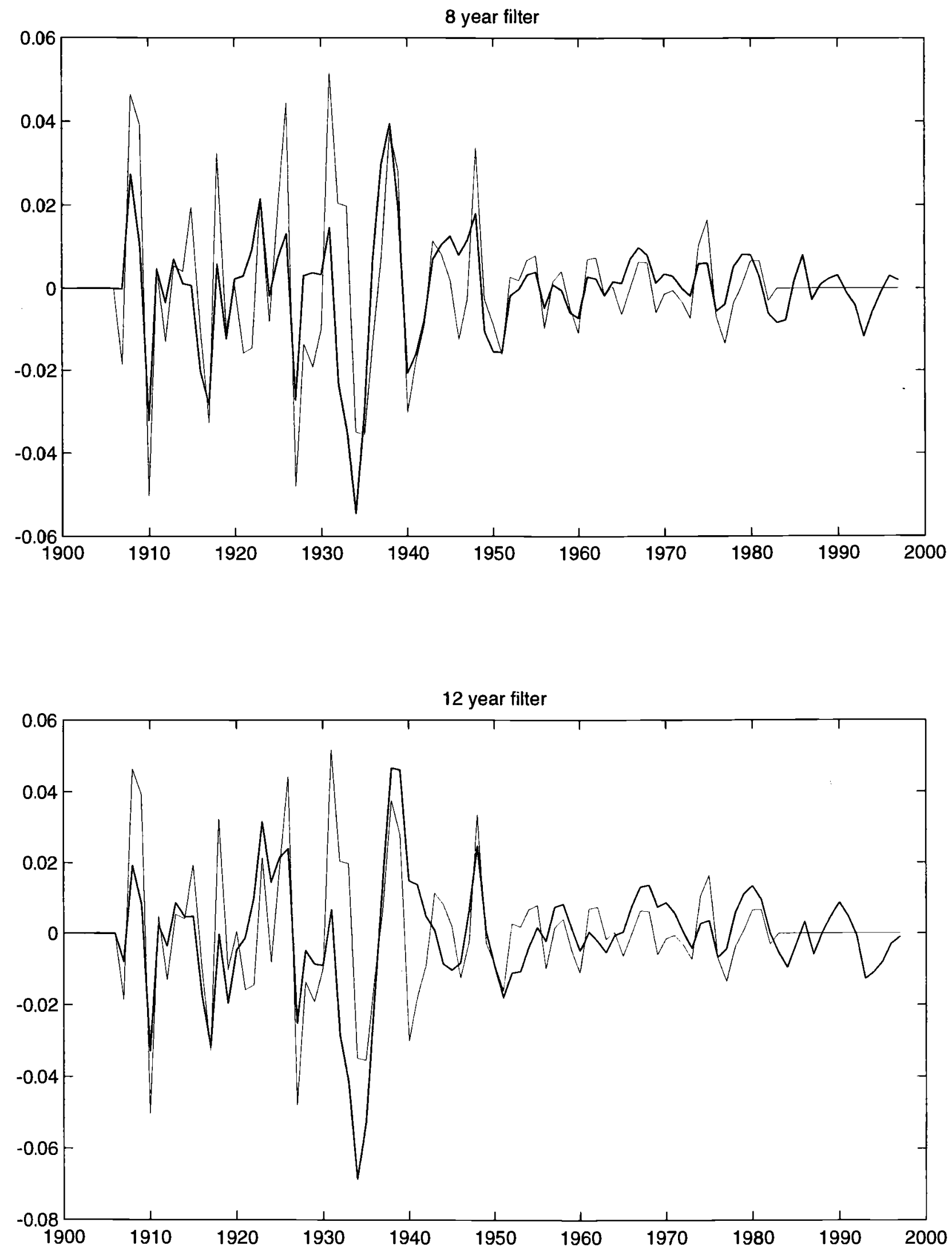

(The thin line is obtained with a two-sided, 8 year filter) 
Figure 3B Consumption Deviations from Trend

16 year filter

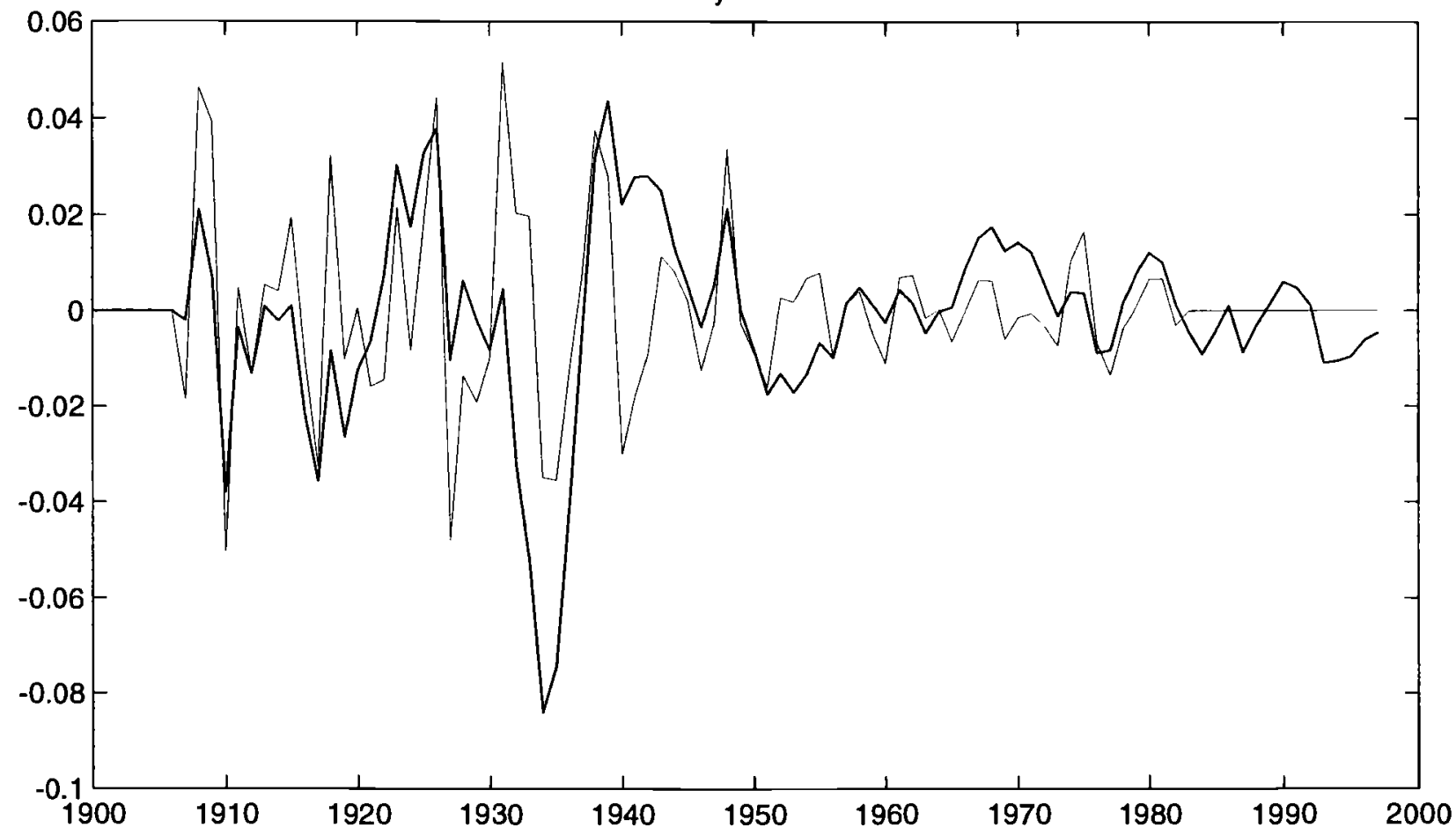

20 year filter

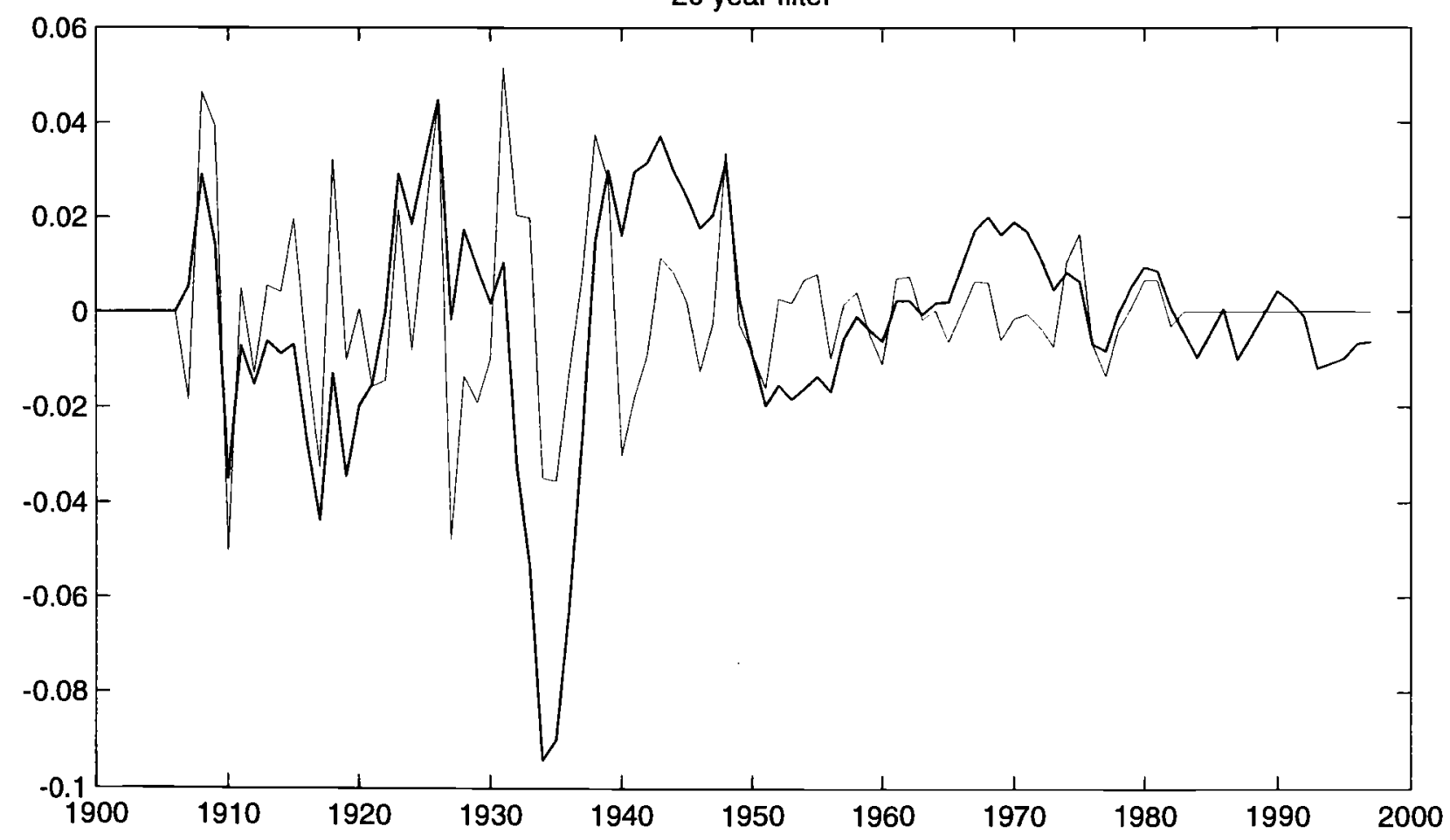

(The thin line is obtained with a two-sided, 8 year filter) 
Figure 3C Consumption Deviations from Trend
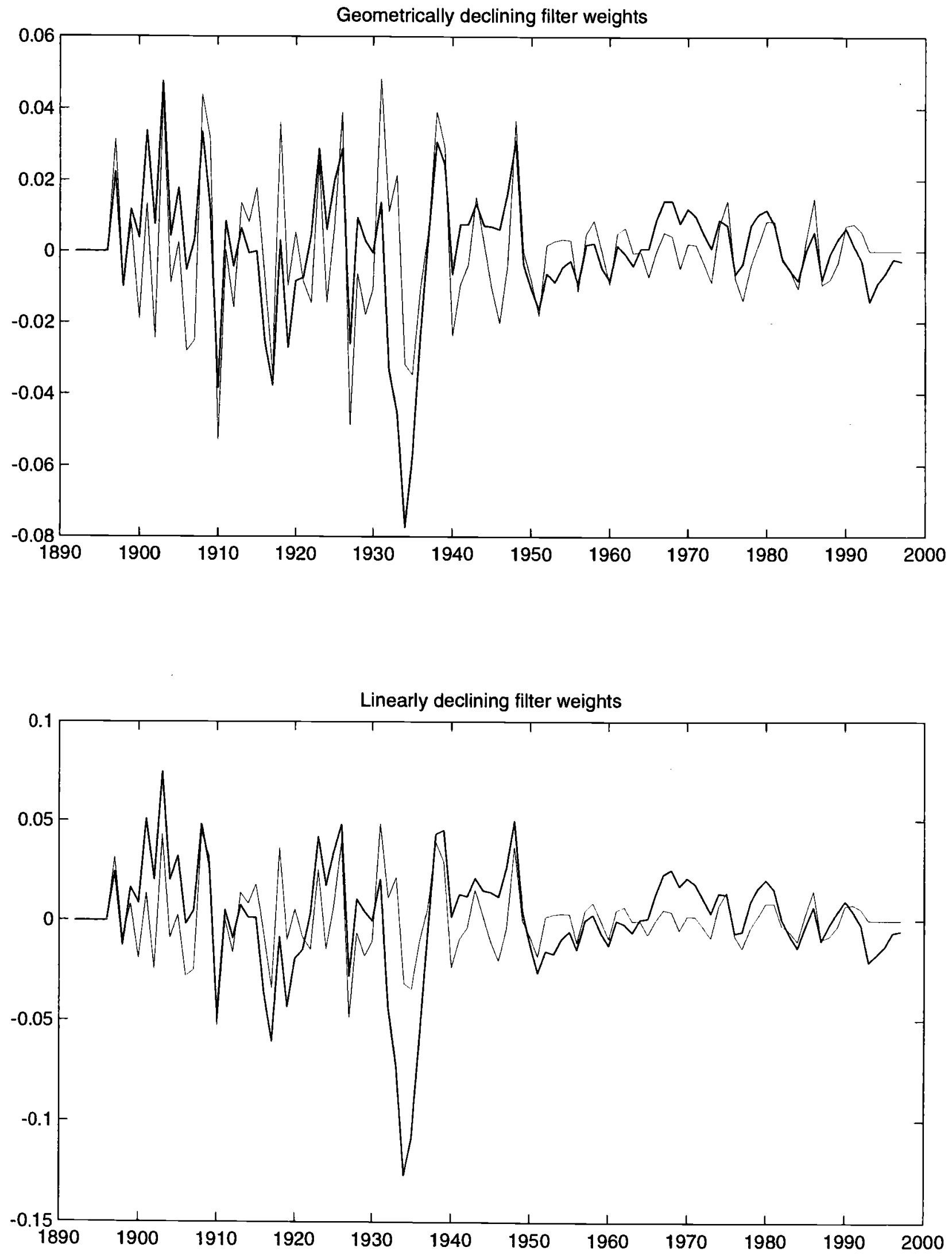

(The thin line is obtained with a two-sided, 8 year filter) 
Figure $4 \quad$ Transfer function for adjusted one-sided filter (8years, theta $=1.4$ )

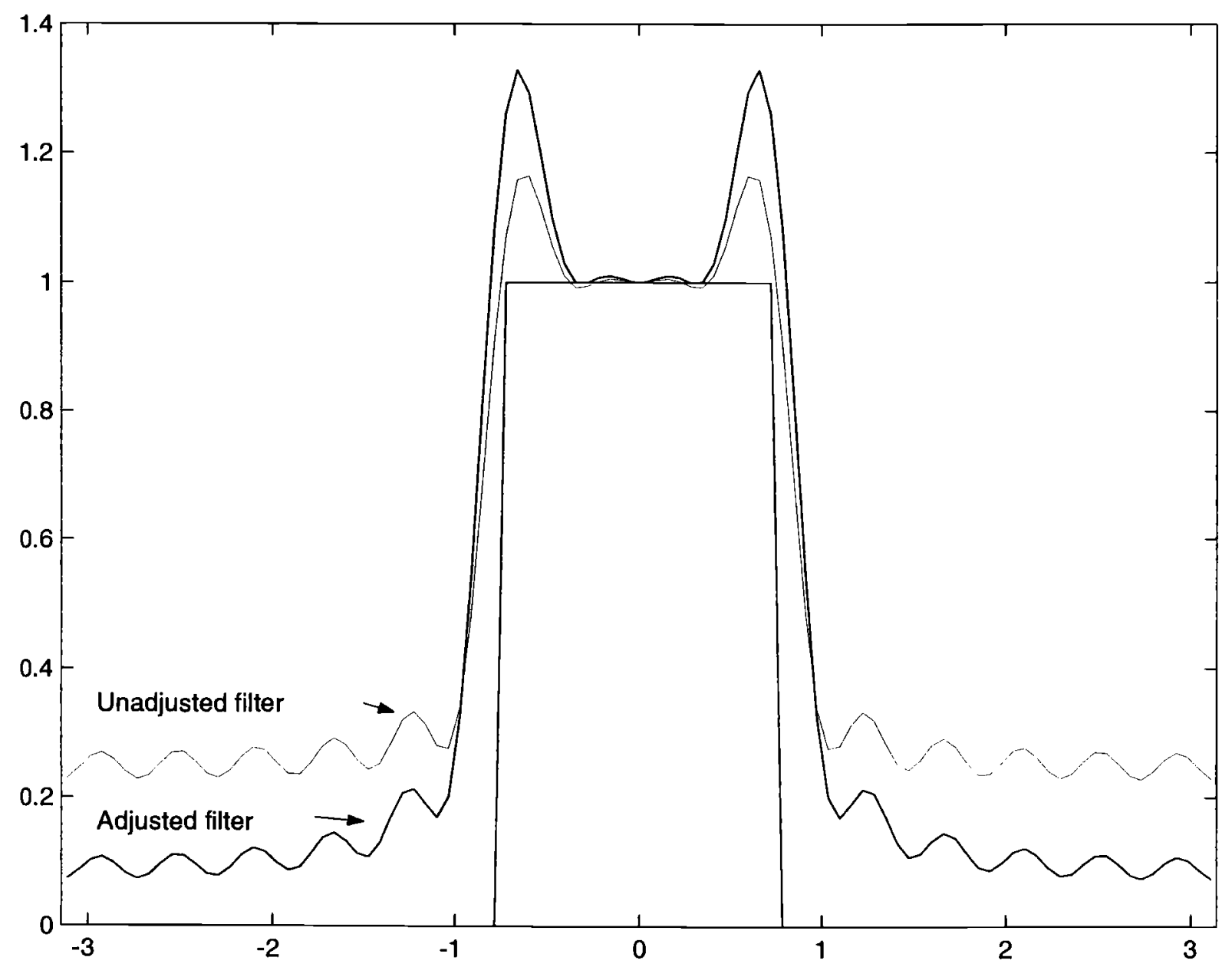

